\title{
Zeeman line formation in solar magnetic fields
}

\section{Studies with empirical probability distribution functions}

\author{
M. Sampoorna ${ }^{1,2, \star}$, K. N. Nagendra ${ }^{1,2}$, H. Frisch ${ }^{2}$, and J. O. Stenflo ${ }^{3}$ \\ 1 Indian Institute of Astrophysics, Koramangala, Bangalore 560 034, India \\ e-mail: sampoorna@iiap.res.in \\ 2 Laboratoire Cassiopée, CNRS, Université de Nice, Observatoire de la Côte d'Azur, BP 4229, 06304 Nice Cedex 4, France \\ 3 Institute of Astronomy, ETH Zürich, 8092 Zürich, Switzerland
}

Received 28 November 2007 / Accepted 11 April 2008

\section{ABSTRACT}

\begin{abstract}
Context. Numerical simulations of magneto-convection and analysis of solar magnetograms provide probability distribution functions (PDFs) for the magnetic field strength.

Aims. In the paper, we explore the effects of these PDFs on Zeeman line formation.

Methods. We calculate the mean Stokes parameters for a Milne-Eddington atmosphere in the limit of optically thin (micro-turbulent) and thick (macro-turbulent) magnetic structures and also the dispersion around the mean profiles in the optically thick limit. Several types of PDFs are considered: (a) Voigt function and stretched exponential type PDFs for fields with fixed direction but fluctuating strength; (b) a cylindrically symmetrical power law for the angular distribution of magnetic fields with given field strength; (c) composite PDFs accounting for randomness in both strength and direction obtained by combining a Voigt function or a stretched exponential with an angular power law. For optically thin structures, explicit expressions are given for the mean values of the Zeeman absorption matrix elements. We also describe how the averaging technique for a normal Zeeman triplet may be generalized to the more common case of anomalous Zeeman splitting patterns.

Results. We show that, for magnetic field rms fluctuations of the order of $6 \mathrm{G}$, consistent with observational data, Stokes $I$ is essentially independent of the shapes of the PDFs but Stokes $Q, U$, and $V$ and also the dispersion around the mean values are quite sensitive to the tail behavior of the PDF. We confirm a previous result that Stokes $V$ is less sensitive to the scale of the magnetic structures than Stokes $Q$ and $U$. The composite PDF proposed for the fluctuations of the magnetic field vector has an angular distribution peaked about the vertical direction for strong fields, and is isotropically distributed for weak fields; it can be used to mimic solar surface random fields.
\end{abstract}

Key words. line: formation - polarization - magnetic fields - turbulence - radiative transfer - Sun: atmosphere

\section{Introduction}

Magneto-convection on the Sun has a size spectrum that spans several orders of magnitudes and hence develops turbulent elements or eddies the sizes of which are much smaller than the spatial resolution of current spectro-polarimeters (about 0.2 arcsec or $150 \mathrm{~km}$ at the photospheric level). Thus the Stokes profiles that we observe are always averages, the averaging being over space, time, and along the line of sight. This suggests that it may be sufficient to characterize the magnetic field responsible for spectral line polarization by a probability distribution function (PDF). Attempts have been made to deduce such PDFs from observational data by inversion methods (cf. Domínguez Cerdeña et al. 2006). Here we consider the forward method, namely the calculation of the mean Stokes parameters for a given PDF. Our primary goal is to compare Stokes profiles calculated with different types of PDFs. We have used PDFs determined from observations (Stenflo \& Holzreuter 2002, 2003a,b) and from numerical simulations of magneto-convection (Stein \& Nordlund 2006; Vögler et al. 2005, and the papers cited therein). The observational PDFs have been deduced from magnetograms and

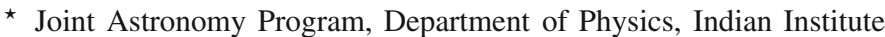
of Science, Bangalore 560 012, India.
}

describe the vertical magnetic field in the solar photosphere. For simplicity we neglect any depth-dependence of the PDFs inside the atmosphere.

In the solar photosphere the mean free path of optical photons is in the range $50-100 \mathrm{~km}$, corresponding approximately to the middle part of the turbulent spectrum. The calculation of mean Stokes parameters for this spatial range, sometimes referred to as meso-turbulence, has been considered by a few authors (Landi Degl'Innocenti 1994; Frisch et al. 2006a,b; Carroll \& Staude 2003, 2005a,b, 2006; Carroll \& Kopf 2007). The mean Stokes parameters for meso-turbulence are always bounded by the micro and macro-turbulent limits. The concept of microturbulence is associated to optically thin magnetic eddies while macro-turbulence corresponds to optically thick ones. In the following we use the much more general terms optically thin and thick limits, since they can also be applied to magnetic fields that are not "turbulent" in the sense of having a nearly isotropic angular distribution but can even be unidirectional. We emphasize that the terminology of "optically thin/ thick" applies to the scale of magnetic field fluctuations and not to the spectral line itself.

In the optically thin limit the Zeeman absorption matrix (containing both absorptive and magneto-optical effects) can be averaged over the PDF and the line transfer equation solved with 
the averaged absorption matrix. Distributions of optically thin elements and transfer equations with mean coefficients were first considered by Stenflo (1971, and references cited therein). One of the effects of optically thin magnetic eddies is to produce line broadening. The earliest observational attempt to search for magnetic fields by this broadening mechanism dates back to Unno (1959). Stenflo \& Lindegren (1977) performed a statistical analysis of 402 unblended Fe I lines in the optical region and found an upper limit of $100 \mathrm{G}$ for the rms magnetic field fluctuations responsible for the line broadening. Along the same lines, Sánchez Almeida et al. (1996) introduced the MISMA model (MIcro Structured Magnetic Atmospheres) consisting of spatially intermittent optically thin structures.

In the optically thick limit, the emergent spectrum may be formed within a single magnetic structure. The averaging over different realizations of the vector magnetic field is now performed on the emergent solution of the transfer equation. Multicomponent models representing optically thick unresolved structures were introduced by Stenflo $(1971,1973,1994)$ and Stenflo et al. (1984). The special case of a two-component model forms the basis of the line ratio technique (Stenflo 1973).

For our present investigation of the effects of various types of PDF we consider both the optically thin and thick limits. The case of Gaussian PDFs was studied by Dolginov \& Pavlov (1972); Domke \& Pavlov (1979); Frisch et al. (2005, 2006a,b, 2007); Sampoorna et al. (2008). Here we use more realistic types of PDFs, namely Voigt type and stretched exponential type distributions for the strength of a magnetic field with a fixed direction. We also consider axially symmetric magnetic fields of constant strength but with random directions distributed according to a power law. Finally we introduce a heuristic PDF, which combines distributions in strength and direction. The angular distribution is described by a power law and the strength distribution by either a Voigt function or a stretched exponential.

The PDFs that we are adopting can be used in any numerical solution of the polarized line transfer equations. Here we use the Unno-Rachkovsky solution (see Unno 1956; Rachkovsky 1962a,b). We believe that the approximation of a MilneEddington (ME) atmosphere is adequate for exploratory work of the sensitivity of Stokes profiles to different magnetic field PDFs. In the optically thin limit we average the Zeeman absorption matrix over the PDF before applying the Unno-Rachkovsky solution. In the optically thick limit we average the UnnoRachkovsky solution over the magnetic field PDF. In these two limits, it is possible within the framework of the ME model to introduce fluctuations of the atmospheric parameters (velocity, temperature, density) and correlations between them and with the magnetic field. Adequate joint PDFs would have to be introduced. Correlations between velocity fields and magnetic fields will produce asymmetric profiles (see e.g. Landi Degl'Innocenti \& Landolfi 2004, Figs. 9.19 and 9.20). Here we analyze only pure magnetic fields effects.

In Sects. 2-5 we present mean Stokes profiles for a Zeeman triplet computed with different kinds of PDFs, for optically thin and optically thick eddies. We also consider the dispersion of the Stokes profiles around their mean values. In Sect. 6 we generalize our averaging procedures to a anomalous Zeeman pattern. Conclusions are presented in Sect. 7.

\section{Magnetic field strength distribution: Voigt type PDF}

Recently Stenflo \& Holzreuter (2002, 2003a,b) have found from an analysis of high resolution La Palma and MDI solar magnetograms that the PDF for the line of sight (LOS) component of the magnetic field is nearly independent of the spatial scale and can be well represented by a simple function which has a Gaussian core and Lorentzian type wings. The Gaussian core is centered around zero field. The PDF wings are generally significantly different for the positive and negative polarities. Stenflo \& Holzreuter $(2002,2003$ a) have proposed a PDF for the LOS field strength that can be represented by a Voigt function depending on a magnetic damping parameter $a_{B}$ and a magnetic width $\Delta_{B}$. The parameter $\Delta_{B}$ is a measure of the rms fluctuations of the LOS component of the field. It represents the width of the Gaussian core, while the parameter $a_{B}$ describes the damping of the Lorentzian wings. These two parameters are not based on any theory but only define a convenient and compact analytical fit function.

If one chooses $\Delta_{B}=6 \mathrm{G}$ and $a_{B}=1.5$, it surprisingly well describes the empirical PDF for the LOS magnetic field strength derived from magnetograms. A symmetric Voigt function however has zero net flux, whereas in real magnetograms the magnetic flux is generally unbalanced locally (although the global ensembles should in general be balanced). Such situations can be well represented by a Voigt PDF that has a symmetric Gaussian core, but with different damping parameters $a_{B}$ for the positive and negative polarities.

We examine in Sect. 2.1 the effects of a symmetric Voigt PDF on the mean Stokes profiles. Because of the symmetry around zero, the mean magnetic field is zero. In Sect. 2.2 we consider asymmetric PDFs with Gaussian core and Lorentzian wings. The corresponding mean fields and mean Stokes $V$ parameters are now different from zero.

\subsection{Symmetric Voigt type PDF}

In terms of the parameters $a_{B}$ and $\Delta_{B}$, the Voigt PDFs considered in this section have the functional form

$P_{\mathrm{V}}\left(\frac{B}{\Delta_{B}}, a_{B}\right)=\frac{a_{B}}{\pi^{3 / 2}} \int_{-\infty}^{+\infty} \frac{\mathrm{e}^{-\left(B_{1} / \Delta_{B}\right)^{2}}}{\left[\left(B-B_{1}\right) / \Delta_{B}\right]^{2}+a_{B}^{2}} \frac{\mathrm{d} B_{1}}{\Delta_{B}}$

Here $B$ is the magnetic field component along a given direction. We introduce the non-dimensional parameters

$y=\frac{B}{\Delta_{B}}, \quad u=\frac{B_{1}}{\Delta_{B}}, \quad \gamma_{B}=\frac{\Delta_{B}}{B_{\mathrm{D}}}$,

where the quantity $B_{\mathrm{D}}$ is

$\frac{1}{B_{\mathrm{D}}}=g \frac{e}{4 \pi m c} \frac{1}{\Delta v_{\mathrm{D}}}$.

Here $g$ is the Landé factor, $c$ the speed of light, $m$ and $e$ the mass and charge of the electron, and $\Delta v_{\mathrm{D}}$ the frequency Doppler width. Thus $\gamma_{B}$ represents the rms fluctuations $\Delta_{B}$ converted to Zeeman shift in Doppler width units. Equation (1) then takes the form

$P_{\mathrm{V}}\left(y, a_{B}\right)=\frac{a_{B}}{\pi^{3 / 2}} \int_{-\infty}^{+\infty} \frac{\mathrm{e}^{-u^{2}}}{(y-u)^{2}+a_{B}^{2}} \mathrm{~d} u$.

For a magnetic damping parameter $a_{B}=0$, this Voigt PDF reduces to a $1 \mathrm{D}$ Gaussian distribution with zero mean field as considered in Frisch et al. (2005).

The average Zeeman absorption matrix $\langle\hat{\Phi}\rangle$ is given by

$\langle\hat{\Phi}\rangle=\int_{-\infty}^{+\infty} \hat{\Phi}(\boldsymbol{B}) P_{\mathrm{V}}\left(\frac{B}{\Delta_{B}}, a_{B}\right) \frac{\mathrm{d} B}{\Delta_{B}}$. 
This integral can be calculated analytically because it involves the convolution of Voigt or Faraday-Voigt functions with a Voigt PDF. Explicit expressions for these convolutions are given in Eq. (5.65) of Landi Degl'Innocenti \& Landolfi (2004, p. 171). For the average absorption coefficients, they yield

$\left\langle\varphi_{I}\right\rangle=\overline{A_{0}}-\frac{1}{3} \overline{A_{2}}\left(3 \cos ^{2} \gamma-1\right)$,

$\left\langle\varphi_{V}\right\rangle=\overline{A_{1}} \cos \gamma$,

$\left\langle\varphi_{Q}\right\rangle=\overline{A_{2}} \sin ^{2} \gamma \cos 2 \chi$,

$\left\langle\varphi_{U}\right\rangle=\left\langle\varphi_{Q}\right\rangle \tan 2 \chi$,

where

$\overline{A_{0}}=\frac{1}{3} \sum_{q=-1}^{q=+1} \frac{1}{\gamma_{q}} H\left(\bar{x}_{q}, \bar{a}_{q}\right), \quad q=0, \pm 1$,

$\overline{A_{1}}=\frac{1}{2} \sum_{q= \pm 1} q \frac{1}{\gamma_{q}} H\left(\bar{x}_{q}, \bar{a}_{q}\right)$,

$\overline{A_{2}}=\frac{1}{4} \sum_{q=-1}^{q=+1}\left(2-3 q^{2}\right) \frac{1}{\gamma_{q}} H\left(\bar{x}_{q}, \bar{a}_{q}\right), \quad q=0, \pm 1$.

The non-dimensional quantities $\gamma_{q}, \bar{x}_{q}$, and $\bar{a}_{q}$ in Eqs. (7) are

$\gamma_{q}=\sqrt{1+q^{2} \gamma_{B}^{2}}, \quad q=0, \pm 1$,

and

$\bar{x}_{q}=\frac{x}{\gamma_{q}}, \quad \bar{a}_{q}=\frac{\left(a+q^{2} \gamma_{B} a_{B}\right)}{\gamma_{q}}$.

In Eq. (9), $x=\left(v-v_{0}\right) / \Delta v_{\mathrm{D}}$ is the frequency measured from the line center in units of the Doppler width $\Delta v_{\mathrm{D}}$, while $a$ is the damping parameter. In the set of Eqs. (6) and (7), $(\gamma, \chi)$ define the orientation of the random magnetic field with respect to the LOS and $H\left(\bar{x}_{q}, \bar{a}_{q}\right)$ is the Voigt function. Because $\bar{x}_{q}$ and $\bar{a}_{q}$ only depend on $q^{2}$, we have $\bar{A}_{1}=0$ and hence $\left\langle\varphi_{V}\right\rangle=0$. For the magneto-optical coefficients $\left\langle\chi_{Q, U, V}\right\rangle$, one has similar expressions, but with Faraday-Voigt functions $F\left(\bar{x}_{q}, \bar{a}_{q}\right)$.

It is important to note that in practical computations with Eq. (5) one should restrict the integration to the range $\left[-B_{\max },+B_{\max }\right]$, where $B_{\max }$ is approximately $1500 \mathrm{G}$. Smallscale photospheric fields with strengths larger than about $1500 \mathrm{G}$ would not be expected to occur, since stronger fields cannot be contained by the ambient gas pressure that prevails in the photosphere. As mentioned above, high resolution La Palma magnetogram data can be represented well with a Voigt like PDF with a magnetic width $\Delta_{B}=6 \mathrm{G}$. Using this as the standard magnetic width, the limits of integration in non-dimensional units are $\left[-y_{\max },+y_{\max }\right]=[-250,+250]$. We have verified that for such a large value of the cut-off, the average absorption matrix (obtained by numerical averaging) does not differ significantly from the corresponding analytical result (see Eq. (6)) derived with $\left[-y_{\max },+y_{\max }\right]=[-\infty,+\infty]$. We have also verified numerically that the part of the PDF that affects the mean absorption coefficient is from $y \approx-50$ to $y \approx+50$. Thus Eq. (6) may indeed be used to analyze the numerical results, to a good approximation. To perform the $y$ integral numerically we use a GaussLegendre quadrature with approximately 300 quadrature points, otherwise one would get oscillations in the wings of the emergent solutions. It has been shown that the maximum frequency band width should be chosen for consistency to satisfy the condition $x_{\max }=4 \gamma_{B} y_{\max }$ (Frisch et al. 2005).
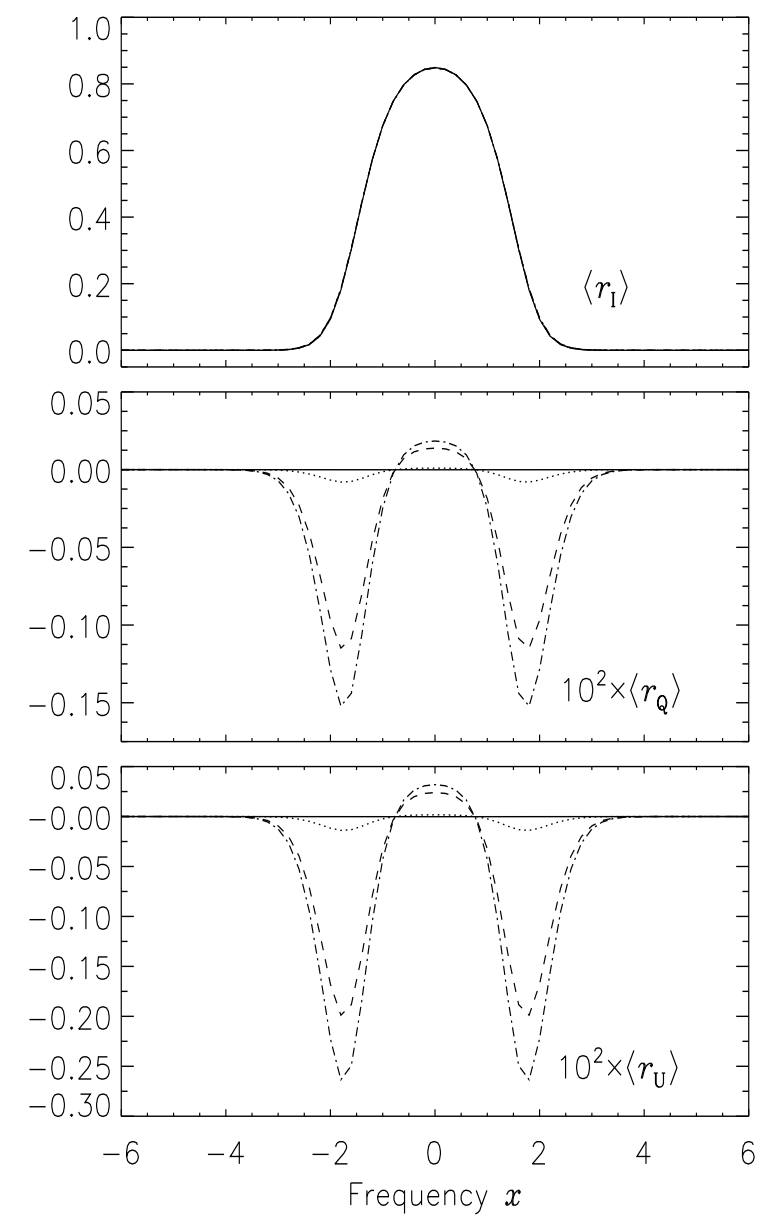

Fig. 1. Effect of the magnetic damping parameter $a_{\mathrm{B}}$ on the mean residual Stokes parameters $\left\langle r_{I, Q, U}\right\rangle$. The average is taken over the Voigt type PDF with zero mean field defined in Eq. (1), assuming optically thin eddies. Different line types correspond to different values of the magnetic damping parameter: $a_{B}=0$ (solid), 0.1 (dotted), 1.5 (dashed), and 2 (dash-dotted). Notice the increase of polarization throughout the line profile, with an increase in $a_{B}$.

We have calculated the averaged emergent residual Stokes parameters $\left\langle r_{I, Q, U}\right\rangle$ for the Voigt type PDF defined in Eq. (1), in the optically thin and optically thick limits. The random field has a fixed orientation with respect to the LOS defined by the polar angles $\gamma=60^{\circ}$ and $\chi=30^{\circ}$. The emergent residual Stokes parameters are defined by $r_{I}=\left[I_{\mathrm{c}}-I\right] / C_{1}$ and $r_{X}=-X / C_{1}$, with the symbol $X$ denoting $Q, U$ or $V$. The constant $C_{1}$ is the slope of the continuum source function $S\left(\tau_{\mathrm{c}}\right)=C_{0}+C_{1} \tau_{\mathrm{c}}$, with $\tau_{\mathrm{c}}$ the continuum optical depth. The continuum intensity at the surface is $I_{\mathrm{c}}=C_{0}+C_{1}$. The model used has damping parameter $a=0$, line strength parameter $\beta=k_{0} / k_{\mathrm{c}}=10$ (ratio of line to the continuous absorption coefficient). We also assume that the spectral line has a wavelength around $5000 \AA$, a Landé factor of 2 and a Doppler width of $1.5 \mathrm{~km} \mathrm{~s}^{-1}$. For this typical line, $B_{\mathrm{D}}=1.07 \times 10^{3} \mathrm{G}$. Hence the value of $\gamma_{B}$ corresponding to rms magnetic field fluctuations $\Delta_{B}=6 \mathrm{G}$ is $\gamma_{B}=0.0056$. This means that we will be dealing here with very small Zeeman shifts. As a result the $\left\langle r_{I}\right\rangle$ profiles in all the figures in this paper remain insensitive to the parameters of the PDFs.

The results presented in Fig. 1 correspond to the optically thin limit. They are calculated with $\gamma_{B}=0.0056$ and $a=0$. The magnetic damping parameter $a_{B}$ is taken as a free parameter. 


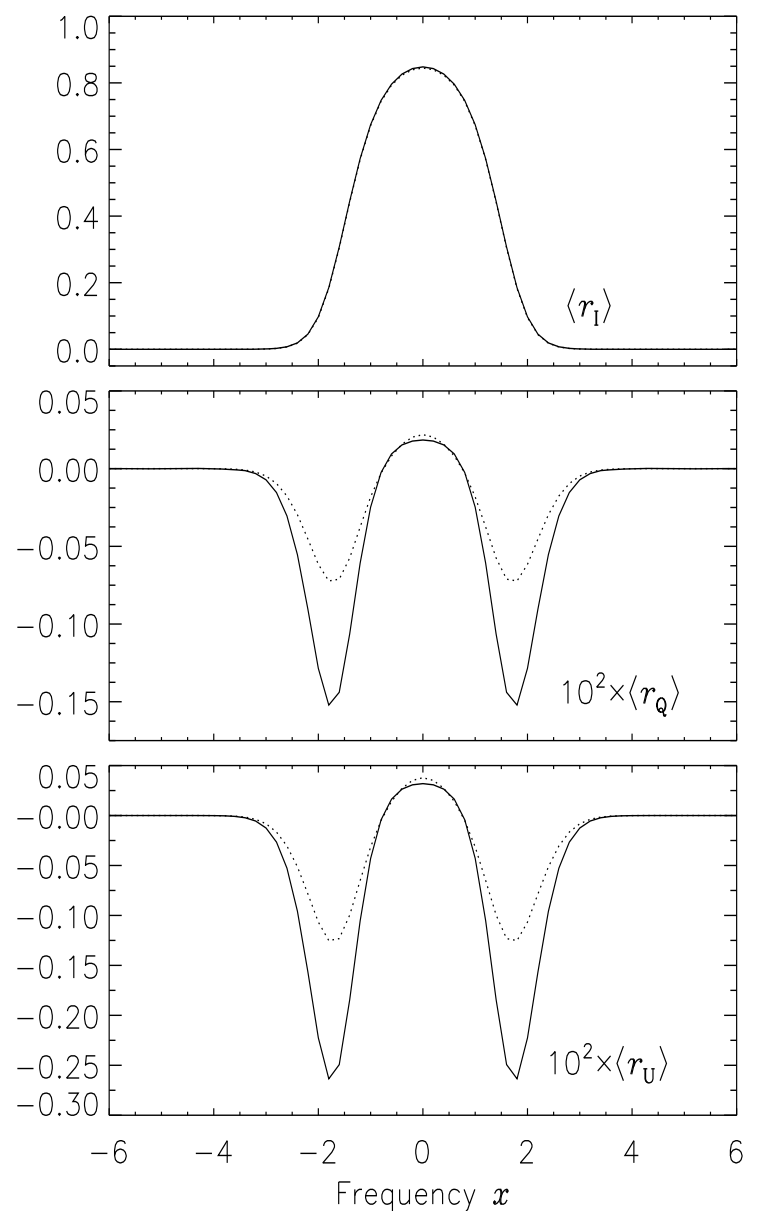

Fig. 2. Comparison of lines formed for optically thin (solid line) and optically thick (dotted line) magnetic eddies. The model parameters are the same as in Fig. 1, but with $a_{B}=1.5$.

Because of the symmetry of the $\operatorname{PDF}\left\langle\varphi_{V}\right\rangle=0$ and hence $\left\langle r_{V}\right\rangle=$ 0 . The $\left\langle r_{I}\right\rangle$ profile is insensitive to the value of $a_{B}$ for the reason given above. The $\left\langle r_{Q, U}\right\rangle$ can be understood from Eqs. (6)-(9). Since $\gamma_{B} \ll 1$, we have $\gamma_{q} \simeq 1, x_{q} \simeq x$ and $\bar{a}_{1} \simeq \gamma_{B} a_{B} \ll 1$ ( $a_{B}$ is zero or of the order of unity). For $a_{B} \neq 0$, we can expand $H\left(x_{q}, \bar{a}_{1}\right)$ in powers of $\bar{a}_{1}$ (see Mihalas 1978, p. 280). We find $\bar{A}_{2} \simeq-(1 / \pi) \gamma_{B} a_{B}[2 x D(x)-1]$, where $D(x)$ is the Dawson function of real argument. This expression explains the order of magnitude of $\left\langle r_{Q, U}\right\rangle$ and the increase with $a_{B}$, i.e. with the broadening of the magnetic field PDF. For $a_{B}=0$, an expansion of $\bar{A}_{2}$ for small $\gamma_{B}$ yields $\bar{A}_{2} \simeq(1 / 4 \sqrt{\pi}) \gamma_{B}^{2}\left(1-2 x^{2}\right) \exp \left(-x^{2}\right)$. The $\left\langle r_{Q, U}\right\rangle$ are thus of the order of $10^{-6}$.

In Fig. 2 we compare the mean residual Stokes vector corresponding to the optically thin (solid line), and optically thick (dotted line) limits. The model is the same as in Fig. 1, but with a fixed magnetic damping parameter $a_{B}=1.5$. As the value of $\gamma_{B}$ is very small, the $\left\langle r_{I}\right\rangle$ profile for both the cases are nearly identical. Significant differences are observed in the wing peaks of $\left\langle r_{Q, U}\right\rangle$, the polarization being much larger for optically thin eddies than for optically thick ones.

\subsection{Asymmetric Voigt type PDF}

Asymmetric Voigt PDFs can be constructed by choosing different values of the magnetic damping parameter $a_{B}$ for different parts of the PDF while the Gaussian core is kept symmetrical.
Figure 3a shows examples of asymmetric PDFs. The three curves are constructed as follows:

solid line: for $y<-1, a_{B}=0.5$; for $y \geq-1, a_{B}=2.5$;

dotted line: for $y<-1, a_{B}=0.5$; for $-1 \leq y \leq+1$, $a_{B}=1.5 ;$ for $y>1, a_{B}=2.5$;

dashed line: for $y<-1, a_{B}=0.1$; for $-1 \leq y \leq+1$, $a_{B}=1.5$; for $y>1, a_{B}=2.9$.

All of them have been normalized to unity and more or less resemble the PDF for the La Palma magnetogram in Fig. 2 of Stenflo \& Holzreuter (2002).

The asymmetry in $P_{\mathrm{V}}\left(y, a_{B}\right)$ can be measured by the difference $\delta(y)=P_{\mathrm{V}}(y>0)-P_{\mathrm{V}}(y<0)$ shown in Fig. 3b. The mean magnetic field is the average of $y$ over $\delta(y)$. Here we denote it by $y_{0}$. The values corresponding to the examples in Fig. $3 \mathrm{a}$ are: (solid line) $y_{0}=3.4 ;\left(\right.$ dotted line) $y_{0}=2.9 ;($ dashed line $)$ $y_{0}=4.5$.

Figure 4 shows the mean residual emergent solutions for the 3 PDFs in Fig. 3a in the optically thin limit. The other model parameters are the same as in Figs. 1 and 2. The $\left\langle r_{Q, U}\right\rangle$ profiles show a very small sensitivity to the asymmetry of the PDFs and the $\left\langle r_{I}\right\rangle$ profiles remain insensitive to the PDF asymmetry for the reason given above. For all three PDFs, $\left\langle r_{V}\right\rangle$ peaks around $x \approx 1.5$. The amplitude of the peaks increases with the mean field. For the two first cases of asymmetry (solid and dotted lines in Fig. 3), the values of $\left\langle r_{V}\right\rangle$ do not differ significantly as the $y_{0}$ have similar values.

We have computed also the mean Stokes profiles for the optically thick limit using the same three asymmetric PDFs. For $\left\langle r_{I, Q, U}\right\rangle$, the differences between the optically thin and thick limit solutions are essentially similar to those shown in Fig. 2. For $\left\langle r_{V}\right\rangle$ the differences are minimal. An explanation is provided at the end of Sect. 3.

In a random magnetic field, the Stokes profiles fluctuate around their mean values. The order of magnitude of the fluctuations is given by the dispersion (square root of the variance) $\sigma_{X},(X=I, Q, U, V)$. For the reduced Stokes profiles,

$\sigma_{X}^{2}=\left\langle r_{X}^{2}\right\rangle-\left\langle r_{X}\right\rangle^{2}$

where the average \langle\rangle is over all the realizations of the random field. In the optically thin limit, $\sigma_{X}$ is zero since $r_{X}$ is equal to its mean value. The dispersion has its maximum value in the optically thick limit. Then \langle\rangle stands for the average over the UnnoRachkovsky solution. Examples of the variation of $\sigma_{X}$ with the characteristic scale of the magnetic field can be found in Frisch et al. (2006a). Details on the numerical work required to calculate the dispersion in the optically thick limit can be found also in this reference.

It is interesting to consider the dispersion $\sigma_{X}$ for a given value of the mean field $y_{0}$, and to compare it with the spread introduced in $\left\langle r_{X}\right\rangle$ by varying $y_{0}$ (see Fig. 4). In Fig. 5 we show $\left\langle r_{X}\right\rangle \pm \sigma_{X}$, with $X=I, Q, U, V$ for $y_{0}=3.4$. The other values of $y_{0}(2.9$ and 4.5$)$ yield very similar results. The dispersion is very small for $\left\langle r_{I}\right\rangle$ but for $\left\langle r_{Q, U, V}\right\rangle$ much larger than the spread introduced by different choice of $y_{0}$. One should not forget that the values shown in Fig. 5 are upper bounds.

\section{Magnetic field strength distribution: stretched exponential type PDF}

Numerical simulations of magneto-convection near the solar surface by Stein \& Nordlund (2006) show that the magnetic field is 

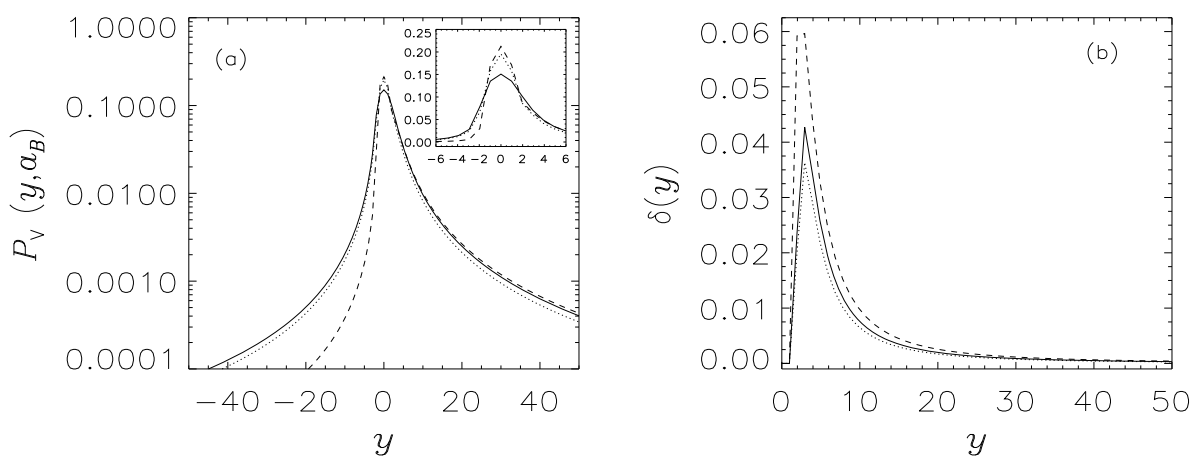

Fig. 3. a) Asymmetric Voigt PDFs defined in Sect. 2.2. A multiplication of the nondimensional unit $y$ by $\Delta_{B}$ yields the magnetic field strength in Gauss. According to Stenflo \& Holzreuter (2002) $\Delta_{B}=6 \mathrm{G}$ in the solar atmosphere. The PDFs are shown in log-scale to resolve the wing region and in linear scale to resolve the core region. b) The asymmetry $\delta(y)$ for the PDFs shown in panel a). In both panels, solid line: $y_{0}=3.4$; dotted line: $y_{0}=2.9$; dashed line: $y_{0}=4.5$.

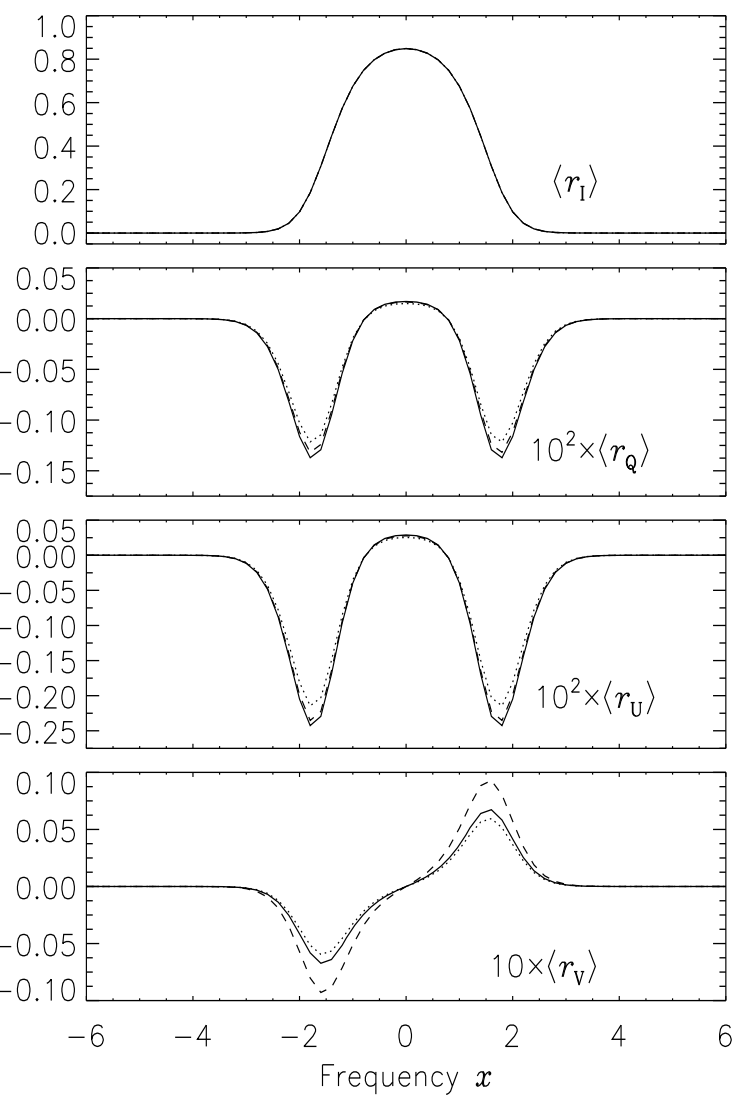

Fig. 4. $\left\langle r_{I, Q, U, V}\right\rangle$ computed with the asymmetric Voigt PDFs in Fig. 3. The relation between line type and PDF is the same as in Fig. 3, namely, solid line: $y_{0}=3.4$; dotted line: $y_{0}=2.9$; dashed line: $y_{0}=4.5$.

intermittent with a stretched exponential distribution. We will now consider the effect of such a distribution function on the emergent mean residual Stokes profiles. The functional form of a stretched exponential (abbreviated "se") may be written as ${ }^{1}$

$P_{\text {se }}(y) \mathrm{d} y=C \mathrm{e}^{-|y|^{k}} \mathrm{~d} y$.

Here $y$ is the magnetic field strength, in non-dimensional units, defined as in Eq. (2). We let $y$ vary from $-y_{\max }$ to $+y_{\max }$. The frequency shifts due to the Zeeman effect (measured in Doppler width units) are $\pm y \gamma_{B}$ where $\gamma_{B}$ is defined in Eq. (2). As in the case of Voigt PDFs, we choose $y_{\max }=250, \Delta_{B}=6 \mathrm{G}$ and $\gamma_{B}=0.0056$. The quantity $k$ is a parameter that can range between 0 and 1 and is referred to as the stretching parameter. $C$ is the normalization constant that is determined numerically

\footnotetext{
${ }^{1}$ http://en.wikipedia.org/wiki/Stretched_exponential
}
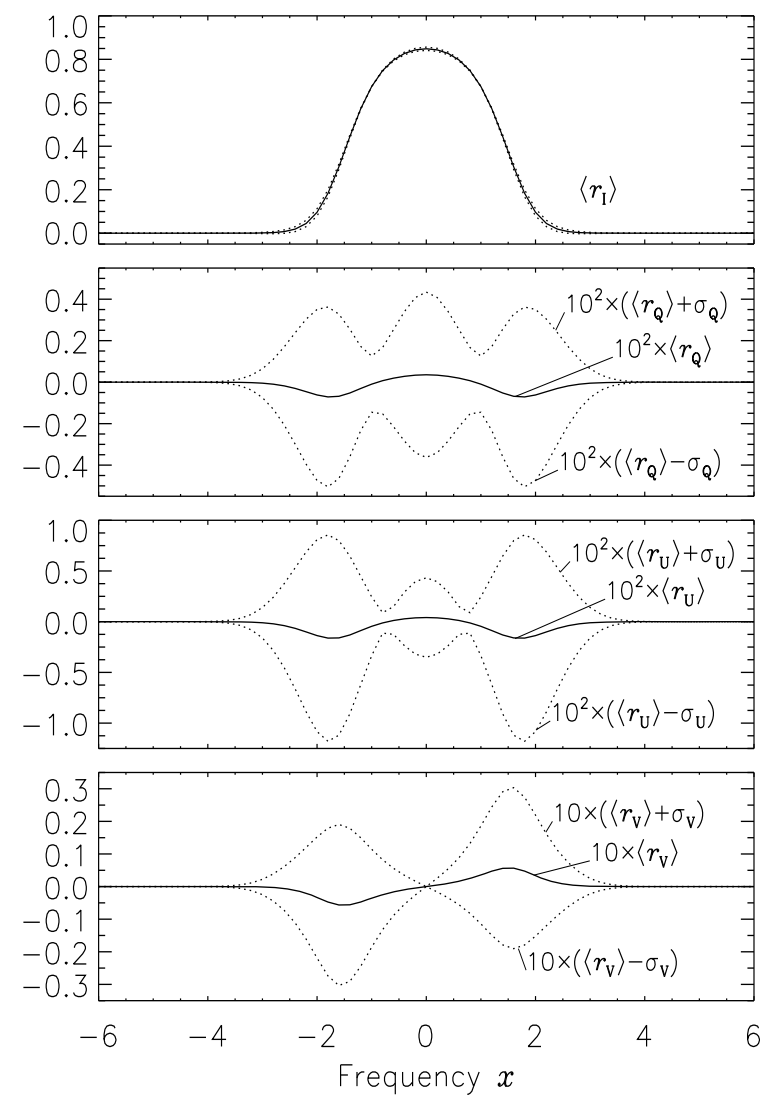

Fig. 5. Dispersion around the mean $\left\langle r_{I, Q, U, V}\right\rangle$ in the optically thick limit. The calculations have been performed with the asymmetric Voigt PDF corresponding to the mean field $y_{0}=3.4$ (see Fig. 3). Notice the fairly large dispersion for $\left\langle r_{Q, U, V}\right\rangle$.

by normalization. For $y_{\max }=\infty, C=k /(2 \Gamma(1 / k))$, where $\Gamma(1 / k)$ is the Gamma function. We find numerically that for $y_{\max }=250$, we have $C \approx k /(2 \Gamma(1 / k))$. The part of the PDF that affects the mean absorption coefficient is from $y \approx-50$ to $y \approx+50$, as above. For accurate evaluation of the $y$ integral we use GaussLegendre quadrature with approximately 300 points.

In Fig. 6 we show stretched exponentials and a Voigt PDF with a damping parameter $a_{B}=1.5$ as suggested by the solar data. The stretched exponentials for $k \leq 0.5$ decrease slower than the Voigt PDF, while for $k>0.5$ they decrease faster. It is clear that the transition at $k_{\text {crit }}=0.5$ depends on the value $a_{B}$. Stenflo \& Holzreuter (2002) mention that Voigt PDFs cannot fit the PDFs derived by magneto-convection simulations. The main problem is the core region, which is much more peaked with stretched exponentials than a Gaussian. 


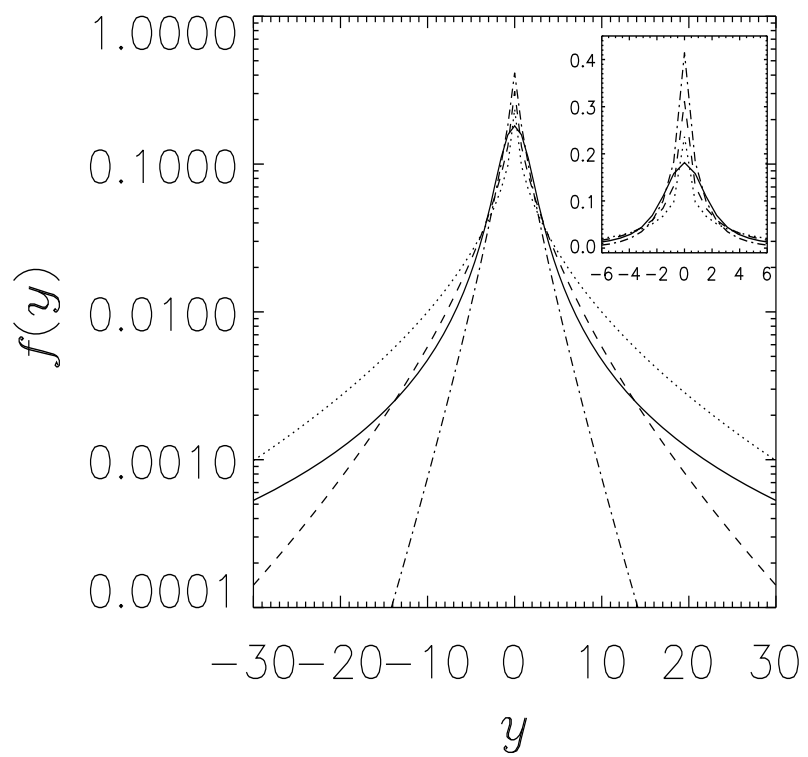

Fig. 6. Stretched exponentials and Voigt PDFs. The non-dimensional $y$-scale is related to the $B$-scale through a multiplying factor $\Delta_{B}$ equal to $6 \mathrm{G}$ according to Stenflo \& Holzreuter (2002). The solid line corresponds to $f(y)=P_{\mathrm{V}}\left(y, a_{B}=1.5\right)$, while the dotted, dashed and dashdotted lines correspond respectively to stretched exponentials $f(y)=$ $P_{\mathrm{se}}(y)$ with the stretching parameter $k=0.5,0.6$, and 0.8 , respectively. The inset figure shows a magnified view of the core region in linear scale.

In Fig. 7 we compare the mean residual Stokes vector for the optically thin limit computed with the stretched exponential PDFs and the Voigt PDF shown in Fig. 6. The Voigt PDF produces the largest degree of linear polarization (cf. $\left\langle r_{Q}\right\rangle$ and $\left\langle r_{U}\right\rangle$ ). As the stretching parameter $k$ increases, the profiles $\left\langle r_{Q, U}\right\rangle$ decrease at all frequencies because of the disappearance of the strong field tails of $P_{\mathrm{se}}(y)$ (see Fig. 6). The $\left\langle r_{I}\right\rangle$ profile as before remains insensitive to the different PDFs.

We find that for a stretched exponential the dispersion and the differences between the optically thin and thick limit solutions are small, much smaller than for the Voigt PDF case, unless the stretching parameter $k$ is chosen to be small $(<0.5)$. Dispersion and differences between the optically thin and thick limits are zero when the magnetic field has a fixed value and orientation. They will remain small as long as the magnetic field PDF shows no extended tails. They will appear with Voigt PDFs or when $k$ is small.

Asymmetric PDFs with non-zero mean field can be constructed with stretched exponentials. It suffices to choose different $k$ values for positive and negative polarities. Figure 8a shows three examples constructed as follows:

solid line: for $y<-1, k=0.8$; for $-1 \leq y \leq+1$, $k=0.7 ;$ for $y>1, k=0.6$;

dotted line: for $y<-1, k=0.9$; for $-1 \leq y \leq+1$, $k=0.7$; for $y>1, k=0.5$;

dashed line: for $y<-1, k=0.8$; for $-1 \leq y \leq+1$, $k=0.6$; for $y>1, k=0.4$.

All these profiles have been normalized to unity. The asymmetries $\delta(y)=P_{\mathrm{se}}(y>0)-P_{\mathrm{se}}(y<0)$ are shown in Fig. $8 \mathrm{~b}$. The values of the mean field $y_{0}$ are: solid line: $y_{0}=1$, dotted line: $y_{0}=3.2$, dashed line: $y_{0}=11.6$.

We have calculated the mean residual Stokes profiles for these three PDFs in the regime of optically thin and optically

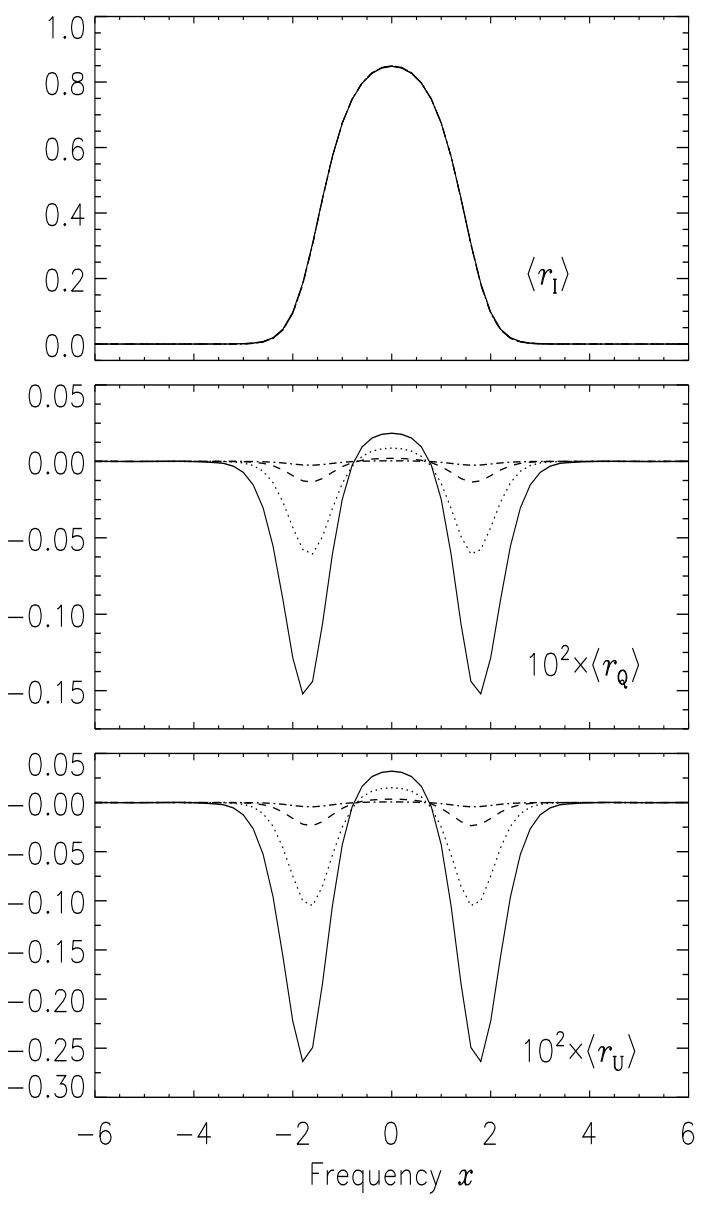

Fig. 7. Mean Stokes profiles $\left\langle r_{I, Q, U}\right\rangle$ for optically thin structures computed using three stretched exponential PDFs with different values of $k$ and one Voigt PDF. The model parameters are the same as in Fig. 2. Line types: solid (Voigt with $\left.a_{B}=1.5\right)$, dotted $(k=0.5)$, dashed $(k=0.6)$, and dash-dotted $(k=0.8)$.

thick magnetic structures. Now $\left\langle r_{V}\right\rangle$ is not zero. Figure 9 shows the results of our calculations for the optically thin limit and one case of optically thick limit corresponding to $y_{0}=11.6$. When $y_{0}=1$ and $y_{0}=3.2$ there are no significant differences between the optically thin and thick solutions because of the rapid drop of the PDF tails.

In the optically thin limit, we observe a clear increase in the peak amplitudes of $\left\langle r_{Q, U, V}\right\rangle$ when the mean field $y_{0}$ increases. The positions of the peaks are however essentially insensitive to the mean field value. The $\left\langle r_{I}\right\rangle$ profiles show no splitting and remain insensitive to the changes in the asymmetries. Differences between the optically thin and thick limits appear for Stokes $Q$ and $U$ when $y_{0}=11.6$ and are due to the extended tail of the PDF for positive polarities. For Stokes $V$, the differences remain small even for $y_{0}=11.6$. The relative insensitivity of Stokes $V$ to the scale of the magnetic field fluctuations has been observed for Voigt type PDFs (see Sect. 2.2) and also for symmetric Gaussian PDFs with non-zero mean field (Frisch et al. 2006a). It is due to the fact that the function $\delta(y)$ which measures the imbalance between positive and negative polarities (see Figs. 3b and $8 b$ ) is sharply peaked around the mean magnetic field value. In the limit of a Dirac distribution, there would be no difference between the optically thin and thick limits since the magnetic field would be deterministic. It thus appears that the mean value of Stokes $V$ can be calculated with reasonable confidence using the optically thin limit, a remark that was already made in 

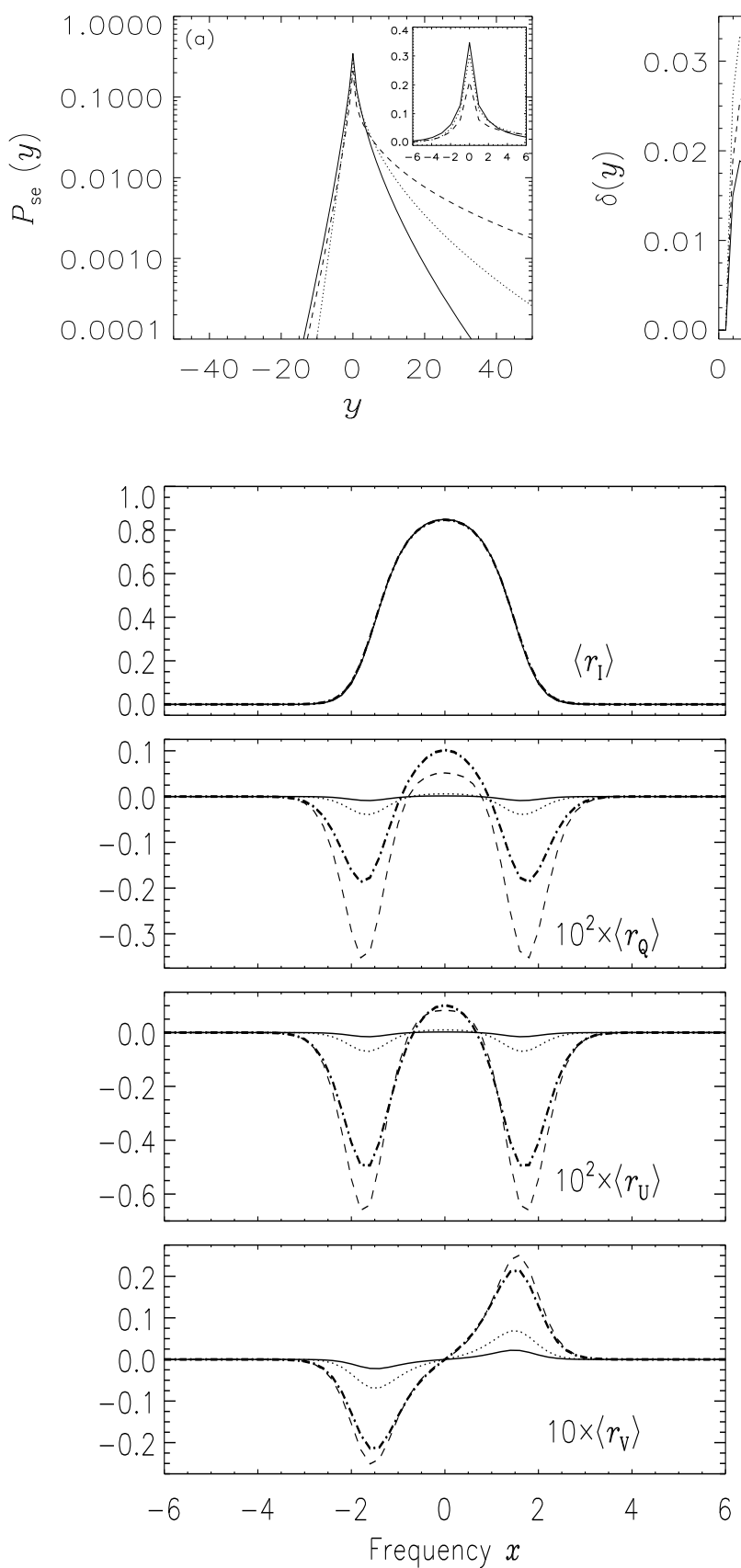

Fig. 9. Mean Stokes profiles $\left\langle r_{I, Q, U, V}\right\rangle$ computed using asymmetric stretched exponential PDFs. The solid, dotted and dashed lines are optically thin limit calculations, the line types referring to the corresponding PDFs in Fig. 8a. The heavy dot-dashed line is an optically thick limit calculation for $y_{0}=11.6$.

Frisch et al. (2006a). Finally we recall that differences between the optically thin and thick limits can occur only for reasonably strong lines $(\beta \gg 1)$ for which transfer effects are significant.

We have calculated the dispersion around the mean Stokes parameters (for the optically thick limit). In Fig. 10, we show the dependence of $\sigma_{V}$ on the value of $y_{0}$. For $y_{0}=11.6$, it can become as large as the dispersion for a Voigt PDF shown in Fig. 5. The dependence of $\sigma_{I, Q, U}$ on $y_{0}$ is similar to that of $\sigma_{V}$, and hence we do not present them here.
Fig. 8. a) Asymmetric stretched exponential PDFs defined in Sect. 3. b) The asymmetry $\delta(y)$ of $P_{\mathrm{se}}(y)$ for the PDFs shown in panel a). In panels a) and b) the line types are solid line: $y_{0}=1$, dotted line: $y_{0}=3.2$, dashed line: $y_{0}=11.6$. To convert to the field strength scale, multiply $y$ by $\Delta_{B}=6 \mathrm{G}$.

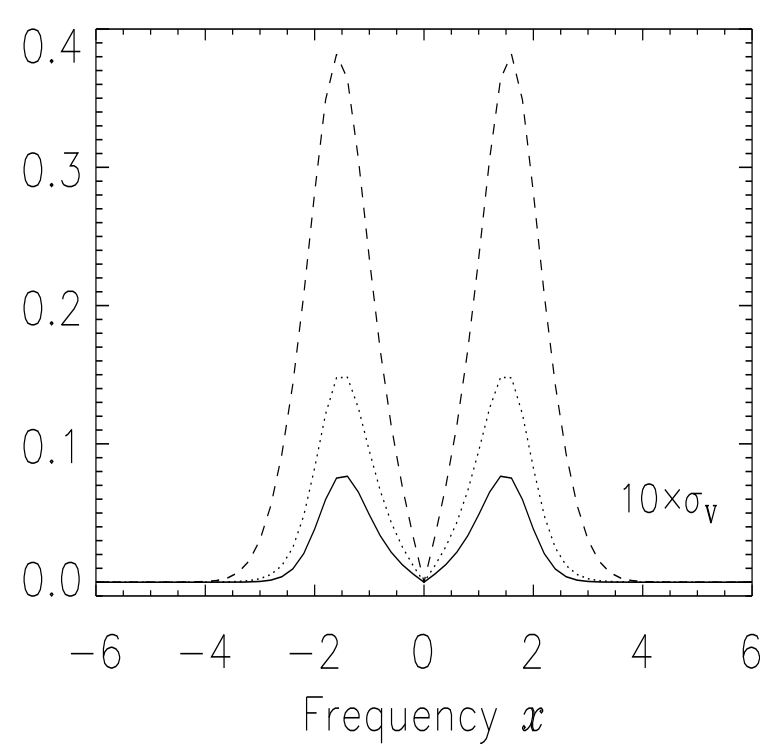

Fig. 10. Dispersion $\sigma_{V}$ in the optically thick limit for the model of Fig. 9. Different line types are solid line: $y_{0}=1$, dotted line: $y_{0}=3.2$, dashed line: $y_{0}=11.6$.

\section{Magnetic field angular distribution: power law type PDF}

A large fraction of the solar atmosphere is filled with mixed polarity fields, and the inter-granular lanes contain fields directed upward or downward. To represent this scenario, we consider magnetic fields that have a fixed value of the strength $B$ but random orientations. For such a random field, the following angular distribution has been suggested by Stenflo (1987):

$P_{\mathrm{pl}}\left(\mu_{B}\right)=\frac{(p+1)}{4 \pi}\left|\mu_{B}\right|^{p}, \quad-1 \leq \mu_{B} \leq+1$.

Here, $\mu_{B}=\cos \theta_{B}$, with $\theta_{B}$ the field orientation with respect to the vertical direction, chosen as the normal to the atmosphere. The abbreviation "pl" stands for "power law". In Stenflo (1987) the same expression as above is given without modulus on $\mu_{B}$, but it is actually Eq. (12) which is meant.

The power law index $p$ can take any value. The $p=0$ case corresponds to an isotropic distribution. As $p$ increases the distribution becomes more and more peaked in the vertical direction (see Fig. 11). One can verify that the power law $P_{\mathrm{pl}}\left(\mu_{B}\right)$ is normalized to unity i.e.,

$\int_{0}^{2 \pi} \int_{-1}^{+1} P_{\mathrm{pl}}\left(\mu_{B}\right) \mathrm{d} \mu_{B} \mathrm{~d} \phi_{B}=1$, 
where $\phi_{B}$ is the azimuth of the random field. In Eq. (12) $P_{\mathrm{pl}}\left(\mu_{B}\right)$ is expressed in the atmospheric reference frame (ARF). Therefore the Zeeman absorption matrix usually formulated in the LOS frame needs to be transformed to the ARF. The required transformation is given by (cf. Varshalovich et al. 1988; Frisch et al. 2007):

$$
\begin{aligned}
\left(\begin{array}{c}
\sin \gamma \cos \chi \\
\sin \gamma \sin \chi \\
\cos \gamma
\end{array}\right)= & \left(\begin{array}{ccc}
\cos \theta \cos \phi & \cos \theta \sin \phi & -\sin \theta \\
-\sin \phi & \cos \phi & 0 \\
\sin \theta \cos \phi & \sin \theta \sin \phi & \cos \theta
\end{array}\right) \\
& \times\left(\begin{array}{c}
\sin \theta_{B} \cos \phi_{B} \\
\sin \theta_{B} \sin \phi_{B} \\
\cos \theta_{B}
\end{array}\right),
\end{aligned}
$$

where $(\theta, \phi)$ defines the orientation of the LOS with respect to the ARF, $(\gamma, \chi)$ the field orientation with respect to the LOS, and $\left(\theta_{B}, \phi_{B}\right)$ the field orientation with respect to the ARF.

The average Zeeman absorption matrix $\langle\hat{\Phi}\rangle$ is given by

$$
\langle\hat{\Phi}\rangle=\int_{0}^{2 \pi} \mathrm{d} \phi_{B} \int_{-1}^{1} \hat{\Phi}(\boldsymbol{B}) P_{\mathrm{pl}}\left(\mu_{B}\right) \mathrm{d} \mu_{B} .
$$

Using the transformation formula of Eq. (14), it can be shown that

$\left\langle\varphi_{I}\right\rangle=A_{0}-\frac{1}{3} A_{2} \frac{p}{p+3}\left(3 \mu^{2}-1\right)$,

$\left\langle\varphi_{Q}\right\rangle=A_{2} \frac{p}{p+3}\left(1-\mu^{2}\right)$,

where $\mu=\cos \theta$. The coefficients $A_{0,2}$ are given by

$$
\begin{aligned}
& A_{0}=\frac{1}{3} \sum_{q=-1}^{q=+1} H\left(x_{q}, a\right), \quad q=0, \pm 1 \\
& A_{2}=\frac{1}{4} \sum_{q=-1}^{q=+1}\left(2-3 q^{2}\right) H\left(x_{q}, a\right), \quad q=0, \pm 1,
\end{aligned}
$$

where $x_{q}=x-q B / B_{\mathrm{D}}$. We note that $\left\langle\varphi_{I, Q}\right\rangle$ depend on the orientation $\theta$ of the LOS but are independent of azimuth $\phi$. When we use the transformation formula (14), we obtain terms proportional to $\cos \left(\phi-\phi_{B}\right), \sin \left(\phi-\phi_{B}\right), \cos 2\left(\phi-\phi_{B}\right)$, or $\sin 2\left(\phi-\phi_{B}\right)$, which vanish when averaged over $\phi_{B}$. As a result $\left\langle\varphi_{U}\right\rangle=0$. Further, $\left\langle\varphi_{V}\right\rangle=0$ for symmetry reasons. As for the anomalous dispersion coefficients, the only non-zero coefficient is $\left\langle\chi_{Q}\right\rangle$. It has the same expression as $\left\langle\varphi_{Q}\right\rangle$ with the Voigt function replaced by the Faraday-Voigt function. From Eqs. (16) and (17) we see that when $p=0$ (isotropic turbulence case), or when $\mu=1$ (disk center observation), $\left\langle\varphi_{Q}\right\rangle=0$.

The mean profiles $\left\langle r_{I, Q}\right\rangle$ have been calculated in the optically thin and thick limits. The average absorption matrix elements given by Eqs. (16) and (17) have been used for the optically thin limit. The model parameters are magnetic field strength $B / B_{\mathrm{D}}=$ 0.1 , line strength $\beta=10$, damping parameter $a=0$. The index $p$ of the power law PDF is taken as a free parameter.

Figure 12 shows $\left\langle r_{I, Q}\right\rangle$ for the optically thin limit, at the limb $(\mu=0.1)$. The results are fairly simple to explain. Since the magnetic field is weak $\left(B / B_{\mathrm{D}}=0.1\right), x_{q} \simeq x$. As a consequence $A_{2}$ is very small. This implies $\left\langle\varphi_{I}\right\rangle \approx H(x, a)$ (see Eq. (16)). Hence $\left\langle\varphi_{I}\right\rangle$ is independent of $p$. The small value of $A_{2}$ also implies $\left\langle\varphi_{Q}\right\rangle \ll\left\langle\varphi_{I}\right\rangle$ and thus $\left\langle r_{Q}\right\rangle \ll\left\langle r_{I}\right\rangle$. As already mentioned, $\left\langle\varphi_{Q}\right\rangle=0$ when $p=0$, hence $\left\langle r_{Q}\right\rangle$ is also zero. As $p$ gets larger, the factor $p /(p+3)$ in Eq. (17) tends to unity. Therefore $\left\langle\varphi_{Q}\right\rangle$

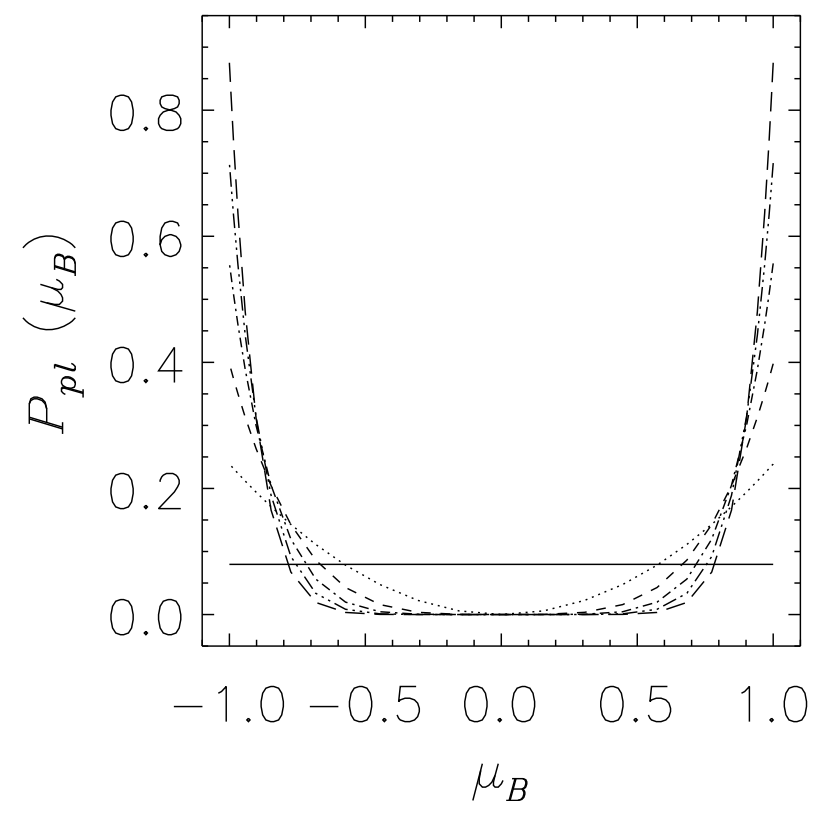

Fig. 11. Power law PDFs as a function of the cosine of the random field orientation $\theta_{B}$ (defined with respect to the vertical). The line types correspond to $p=0$ (solid), 2 (dotted), 4 (dashed), 6 (dash-dotted), 8 (dashtriple-dotted), and 10 (long-dashed). As $p$ increases the PDF becomes more and more peaked in the forward and backward directions.

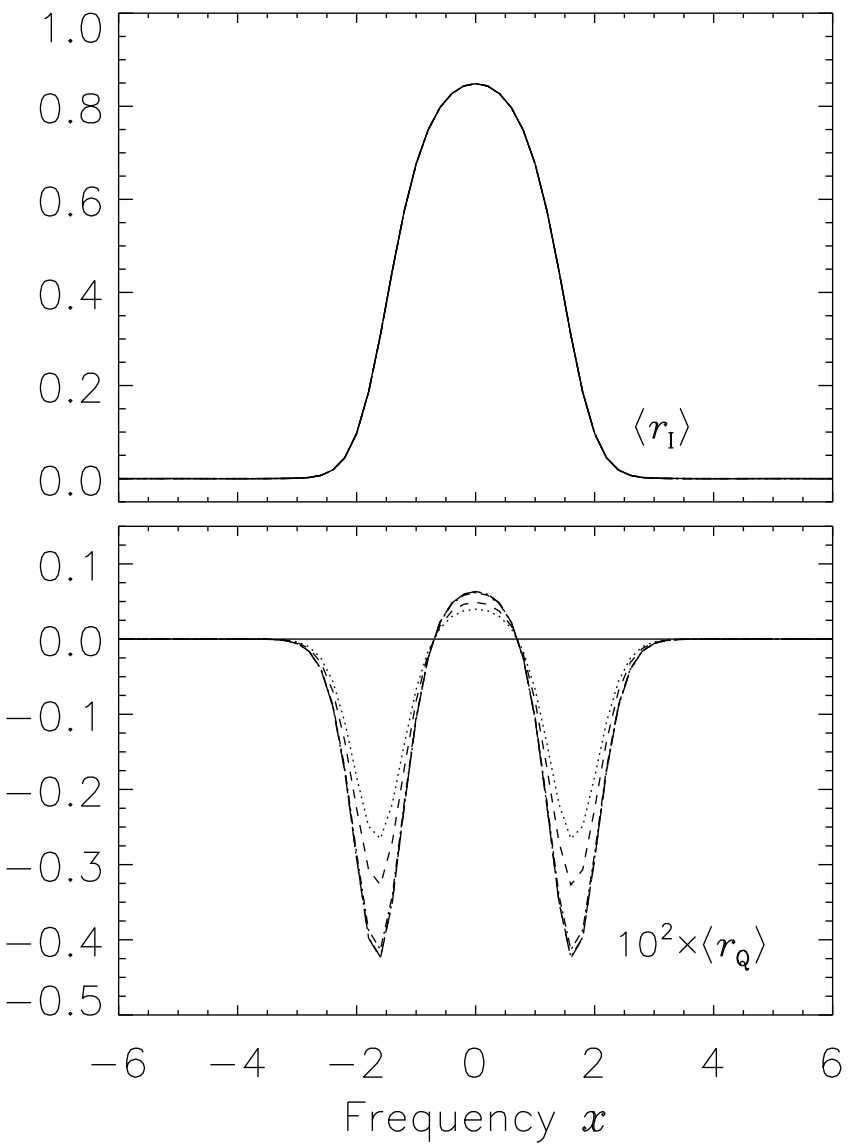

Fig. 12. Residual mean Stokes profiles $\left\langle r_{I, Q}\right\rangle$ at $\mu=0.1$ (limb observation) for the optically thin limit and the angular power law PDF defined in Eq. (12). Line types: $p=0$ (solid), 5 (dotted), 10 (dashed), 100 (dotdashed), 500 (dash-triple-dotted), and 1000 (long dashed). In this case $\left\langle r_{U, V}\right\rangle=0$. 
first increases with $p$ and then saturates. A similar behavior is exhibited by $\left\langle r_{Q}\right\rangle$. The saturation is reached for $p \simeq 100$ as can be observed in Fig. 12.

The mean profiles $\left\langle r_{I, Q}\right\rangle$ for the optically thick limit do not differ significantly from the optically thin limit solutions because the absolute value of the magnetic field along the LOS is bounded by the condition $B / B_{\mathrm{D}}=0.1$. The dispersion around the mean value (for the optically thick limit) decreases with an increase in the value of $p$. This was expected because as $p$ increases the field becomes more and more uni-directional and confined to the two values $\pm 0.1 B_{\mathrm{D}}$.

\section{Vector magnetic field distributions}

For a complete description of turbulent vector magnetic fields one needs a PDF that combines the strength and angular distributions. The strength distributions have been determined empirically (Stenflo \& Holzreuter 2002, 2003a,b) and by simulation (Stein \& Nordlund 2006). Little is known from observations about the angular distribution. A very recent analysis of Hinode data suggests a predominance of very inclined hG fields in the internetwork (Orozco Suárez et al. 2007; Lites et al. 2007, 2008). From physical considerations one can argue that the angular distribution should be strongly field-strength dependent. For the strongest fields the distribution should be peaked around the vertical direction, as the strong fields would tend to have an intermittent flux tube morphology, and the powerful buoyancy forces would push the flux tubes to stand upright (like the stems of lotus flowers that are anchored to the bottom of the lake but float on top). The weakest fields on the other hand would be passively moved and bent by the turbulent fluid motions and get so tangled up that their distribution would be nearly isotropic. The transition between the isotropic and peaked distributions would probably be gradual (possibly around $50 \mathrm{G}$ ).

Based on this scenario we propose here PDFs, that are combinations of two PDFs, one for the angular distribution and one for the field strength distribution. For the angular part we use the power law distribution introduced in Sect. 4, while for the field strength part we consider either a Voigt function (see Sect. 2) or a stretched exponential (see Sect. 3).

\subsection{Stretched exponential $*$ power law PDF}

The functional form of the vector magnetic PDF when we combine a stretched exponential with a power law distribution may be written as

$P\left(y, \mu_{B}, \phi_{B}\right) \mathrm{d} \mu_{B} \mathrm{~d} \phi_{B} \mathrm{~d} y=P_{\text {se }}(y) P_{\mathrm{pl}}\left(\mu_{B}\right) \mathrm{d} \mu_{B} \mathrm{~d} \phi_{B} \mathrm{~d} y$,

where the power law is given by Eq. (12), but now used only for the range $\left[0 \leq \mu_{B} \leq 1\right]$. If we choose the symmetric stretched exponential introduced in Eq. (11) we can write

$$
\begin{aligned}
& P\left(y, \mu_{B}, \phi_{B}\right) \mathrm{d} \mu_{B} \mathrm{~d} \phi_{B} \mathrm{~d} y= \\
& \frac{k}{2 \Gamma(1 / k)} \frac{(p+1)}{2 \pi} \mathrm{e}^{-|y|^{k}} \mu_{B}^{p} \mathrm{~d} y \mathrm{~d} \mu_{B} \mathrm{~d} \phi_{B} .
\end{aligned}
$$

Here $y$ varies in the range $\left[-y_{\max },+y_{\max }\right], \mu_{B}$ in the range $[0,1]$, and $\phi_{B}$ in the range $[0,2 \pi]$. The angular and strength distributions are coupled by letting the power law index $p$ depend on $y$. We have chosen $p=|y| / y_{\mathrm{t}}$ with $y_{\mathrm{t}}=B_{\mathrm{t}} / \Delta_{B}$, where $B_{\mathrm{t}}$ represents the field strength around which the transition between isotropic and peaked distribution occurs. We refer to $y_{\mathrm{t}}$ as the transition field strength. We note that $y_{\mathrm{t}}=\infty$ corresponds to fully isotropic distribution for all field strengths. Gauss-Legendre quadrature is used to perform the integration over $y, \theta_{B}$ and $\phi_{B}$. For the $y$ integration we use 300 quadrature points, and for the $\theta_{B}$ and $\phi_{B}$ integrations we use 30 and 10 points, respectively.

The average Zeeman absorption matrix $\langle\hat{\Phi}\rangle$ is obtained by averaging $\hat{\Phi}(\boldsymbol{B})$ over the PDF defined in Eq. (20). The integration over $\mu_{B}$ and $\phi_{B}$ can be performed analytically to obtain

$$
\begin{aligned}
& \left\langle\varphi_{I}\right\rangle=\left\langle A_{0}\right\rangle-\frac{1}{3}\left\langle A_{2}\right\rangle\left(3 \mu^{2}-1\right), \\
& \left\langle\varphi_{Q}\right\rangle=\left\langle A_{2}\right\rangle\left(1-\mu^{2}\right)
\end{aligned}
$$

After averaging, $\left\langle\varphi_{U}\right\rangle=0$ and $\left\langle\varphi_{V}\right\rangle=0$. The mean coefficients in the above equations are given by

$$
\begin{aligned}
& \left\langle A_{0}\right\rangle=\frac{k}{2 \Gamma(1 / k)} \int_{-y_{\max }}^{+y_{\max }} A_{0}(y) \mathrm{e}^{-|y|^{k}} \mathrm{~d} y, \\
& \left\langle A_{2}\right\rangle=\frac{k}{2 \Gamma(1 / k)} \int_{-y_{\max }}^{+y_{\max }} \frac{|y|}{|y|+3 y_{\mathrm{t}}} A_{2}(y) \mathrm{e}^{-|y|^{k}} \mathrm{~d} y,
\end{aligned}
$$

where $A_{0}(y)$ and $A_{2}(y)$ are given by Eq. (18) with $x_{q}=x-q \gamma_{B} y$ and $\gamma_{B} y=B / B_{\mathrm{D}}$ (see in Eq. (3) the definition of $B_{\mathrm{D}}$ ). Notice that $\left\langle A_{2}\right\rangle=0$ and hence $\left\langle\varphi_{Q}\right\rangle=0$, for $y_{\mathrm{t}}=\infty$.

We can also use in Eq. (19) asymmetric stretched exponentials. In that case $\left\langle\varphi_{V}\right\rangle$ is not zero nor is $\left\langle r_{V}\right\rangle$. We have calculated the mean profiles in the optically thin and thick limits for the distributions shown in Fig. 8a. The model parameters are $\left(a, \beta, \gamma_{B}\right)=(0,10,0.0056)$, LOS perpendicular to the atmosphere $(\mu=1)$. For this $\operatorname{LOS},\left\langle r_{Q, U}\right\rangle=0$ due to symmetry. Figure 13 shows $\left\langle r_{I}\right\rangle$ and $\left\langle r_{V}\right\rangle$ in the optically thin limit. As already observed (see Fig. 9), $\left\langle r_{I}\right\rangle$ is insensitive to the asymmetry of $P_{\mathrm{se}}(y)$ because of the very weak value of $\gamma_{B}$. Figures $13 \mathrm{~b}-\mathrm{d}$ show that the magnitude of $\left\langle r_{V}\right\rangle$ increases with the value of the mean field $y_{0}$ as in Fig. 9. As $y_{\mathrm{t}}$ increases, $\left\langle r_{V}\right\rangle$ profiles approach the isotropic case (solid lines in Figs. 13b-d). As the asymmetry increases we need larger and larger values of $y_{\mathrm{t}}$ to uniformly approach the isotropic limit.

The $\left\langle r_{V}\right\rangle$ profiles for the optically thick limit differ from optically thin limit solutions for $y_{0}=11.6$ only, and the differences remain small (as between the thin dashed and thick dot-dashed lines in Fig. 9).

We have calculated the dispersion $\sigma_{V}$ around $\left\langle r_{V}\right\rangle$ for several values of the mean field $y_{0}$ and of the transition field strength $y_{\mathrm{t}}$. For a given value of $y_{\mathrm{t}}$, the dispersion increases in magnitude with $y_{0}$ as shown in Fig. 10. For a given value of $y_{0}$, there is an increase in $\sigma_{V}$ as the angular distribution becomes more and more anisotropic (decreasing $y_{\mathrm{t}}$ ). Actually the increase of $\sigma_{V}$ follows that of $\left\langle r_{V}\right\rangle$ (see Fig. 13d) and the ratio $\sigma_{V} /\left|\left\langle r_{V}\right\rangle\right|$ remains essentially independent of $y_{\mathrm{t}}$. We stress again that the amplitude of $\sigma_{V}$ will decrease with decreasing sizes of the magnetic structures.

\subsection{Voigt $*$ power law PDF}

Next we consider a composite PDF constructed with a Voigt PDF instead of an stretched exponential. We can use symmetric or asymmetric PDFs. With the symmetric PDF defined in Eq. (1), we have

$$
\begin{aligned}
& P\left(y, \mu_{B}, \phi_{B}\right) \mathrm{d} y \mathrm{~d} \mu_{B} \mathrm{~d} \phi_{B}= \\
& \frac{(p+1)}{2 \pi} P_{\mathrm{V}}\left(y, a_{B}\right) \mu_{B}^{p} \mathrm{~d} y \mathrm{~d} \mu_{B} \mathrm{~d} \phi_{B} .
\end{aligned}
$$

The power law index is chosen as $p=|y| / y_{\mathrm{t}}$ as in Sect. 5.1. The range of variations of $y, \mu_{B}$, and $\phi_{B}$ and the numerical integration 

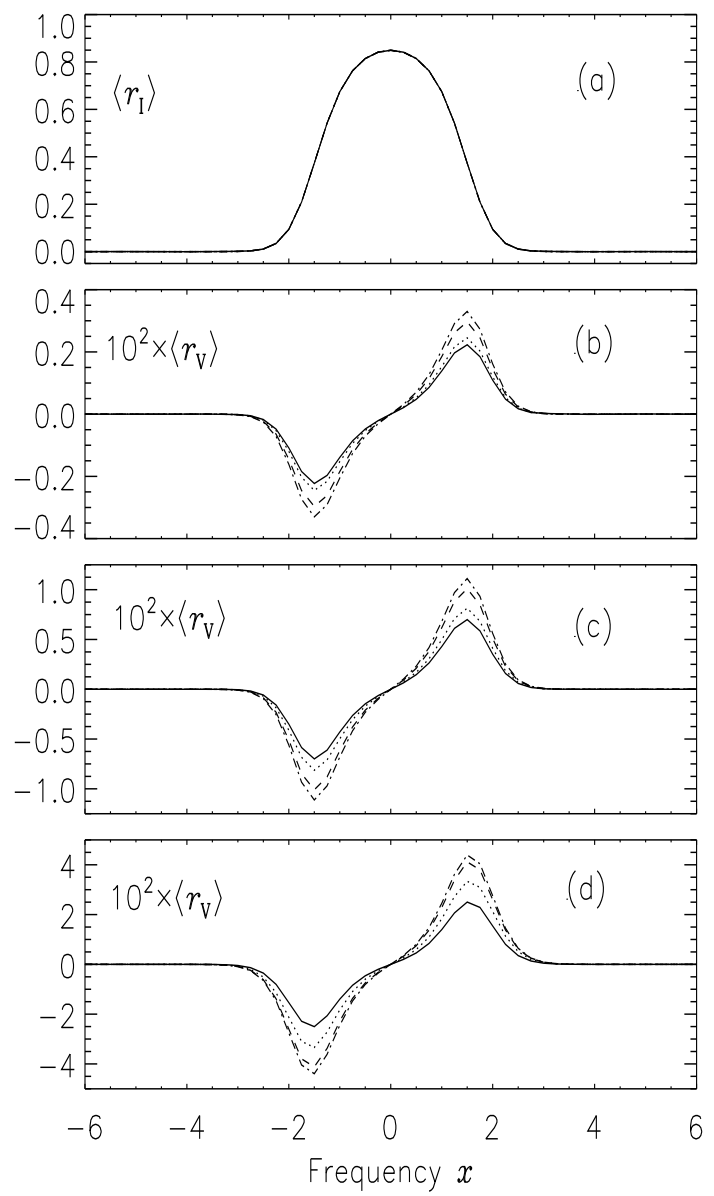

Fig. 13. $\left\langle r_{I, V}\right\rangle$ in the optically thin limit computed using the composite PDF given in Eq. (19) with asymmetric stretched exponential PDFs shown in Fig. 8a. Disk center observation $(\mu=1)$. Line types refer to $y_{\mathrm{t}}=\infty$ (solid), $y_{\mathrm{t}}=50$ (dotted), $y_{\mathrm{t}}=10$ (dashed), and $y_{\mathrm{t}}=5$ (dashdotted). Panels a) and b) correspond to a mean field $y_{0}=1$, panel c) to $y_{0}=3.2$ and panel d) to $y_{0}=11.6$.

scheme are also the same as in Sect. 5.1. The mean coefficients are also given by Eqs. (21)-(24), but with $[k / 2 \Gamma(1 / k)] \mathrm{e}^{-|y|^{k}}$ replaced by $P_{\mathrm{V}}\left(y, a_{B}\right)$.

We have calculated the mean residual Stokes parameters for the asymmetric PDF with mean field $y_{0}=4.5$ (dashed line in Fig. 3a) for the optically thin and thick limits. The model parameters are $\left(a, \beta, \gamma_{B}\right)=(0,10,0.0056)$, the LOS nearly parallel to the limb $(\mu=0.1)$. Figure 14 shows the solutions for optically thin eddies. The different line types correspond to different values of the transition field strength $y_{\mathrm{t}}$. As discussed earlier $\left\langle r_{I}\right\rangle$ is essentially insensitive to the asymmetry of the Voigt PDF and to the variation of $y_{\mathrm{t}}$. When $y_{\mathrm{t}} \rightarrow \infty$, the mean coefficient $\left\langle\varphi_{Q}\right\rangle \rightarrow 0$ since $\left\langle A_{2}\right\rangle \rightarrow 0$ (see Eq. (24)), and hence $\left\langle r_{Q}\right\rangle \rightarrow 0$. As $y_{\mathrm{t}}$ decreases, the PDF becomes more and more peaked and hence $\left\langle r_{Q}\right\rangle$ as well as $\left\langle r_{V}\right\rangle$ increase in magnitude. For symmetry reason, as in Sect. 5.1, $\left\langle\varphi_{U}\right\rangle=0$. However some amount of $\left\langle r_{U}\right\rangle$ is created due to the presence of magneto-optical effects. Therefore $\left\langle r_{U}\right\rangle$ is very small with a behavior similar to $\left\langle r_{Q}\right\rangle$. The differences between the optically thin and thick limit solutions are similar to the one noted in Fig. 2 for $\left\langle r_{I, Q, U}\right\rangle$ and in Fig. 9 for $\left\langle r_{V}\right\rangle$.
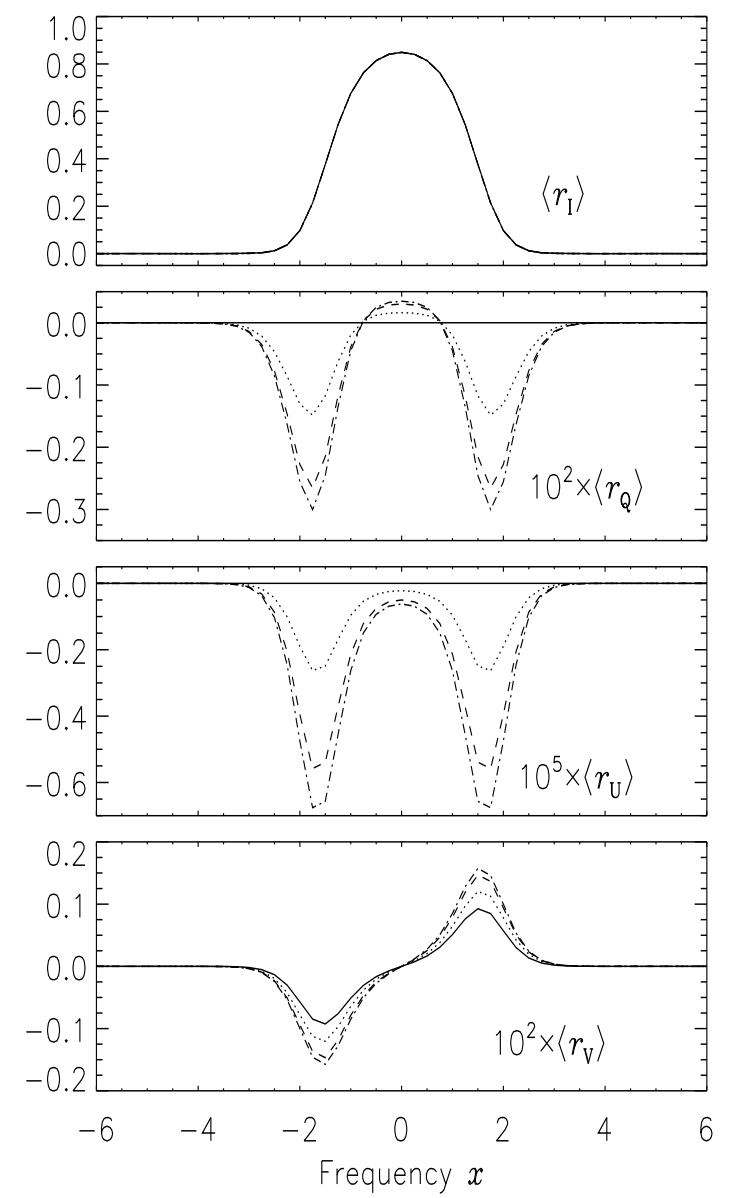

Fig. 14. $\left\langle r_{I, Q, U, V}\right\rangle$ profiles for optically thin limit and for limb observation $(\mu=0.1)$. Composite PDF with asymmetric Voigt function corresponding to a mean field $y_{0}=4.5$ (dashed line of Fig. 3). The line types correspond to $y_{\mathrm{t}}=\infty$ (solid), $y_{\mathrm{t}}=50$ (dotted), $y_{\mathrm{t}}=10$ (dashed), and $y_{\mathrm{t}}=5$ (dash-dotted).

\section{Turbulent line formation for anomalous Zeeman splitting}

For a general Zeeman pattern, the elements of the absorption matrix have contributions from each individual Zeeman component. The relative strengths of these various components is thus needed to calculate the absorption matrix. Under local thermodynamic equilibrium (LTE) the line strengths are proportional to the matrix elements $\left|\left\langle l s j m|\mathbf{D}| l^{\prime} s^{\prime} j^{\prime} m^{\prime}\right\rangle\right|^{2}$, where $\mathbf{D}$ is the dipole operator, and are given by the quantities (see Stenflo 1994, p. 107-111)

$S_{q}\left(M_{1}, M_{\mathrm{u}}\right) \equiv \frac{\tilde{S}_{q}\left(M_{\mathrm{l}}, M_{\mathrm{u}}\right)}{\sum_{M_{\mathrm{l}}, M_{\mathrm{u}}} \tilde{S}_{q}\left(M_{\mathrm{l}}, M_{\mathrm{u}}\right)}, \quad q=0, \pm 1$,

where $M_{1, \mathrm{u}}$ are the magnetic quantum numbers of the lower and upper levels respectively, and $q=M_{1}-M_{\mathrm{u}}$. The unnormalized strengths $\tilde{S}_{q}\left(M_{1}, M_{\mathrm{u}}\right)$ are listed in Table 6.1 of Stenflo (1994).

The Zeeman splitting of the individual components can be written in compact form as

$x_{q}=x-\left[q g_{\mathrm{u}}-M_{\mathrm{l}}\left(g_{\mathrm{u}}-g_{\mathrm{l}}\right)\right] \frac{B}{B_{\mathrm{D}}^{\prime}}$,

where $g_{u, l}$ are the Landé factors of the upper and lower level respectively, and $1 / B_{\mathrm{D}}^{\prime}=e /\left(4 \pi m c \Delta \nu_{\mathrm{D}}\right)$. The elements of the 
(a) Optically thin eddies
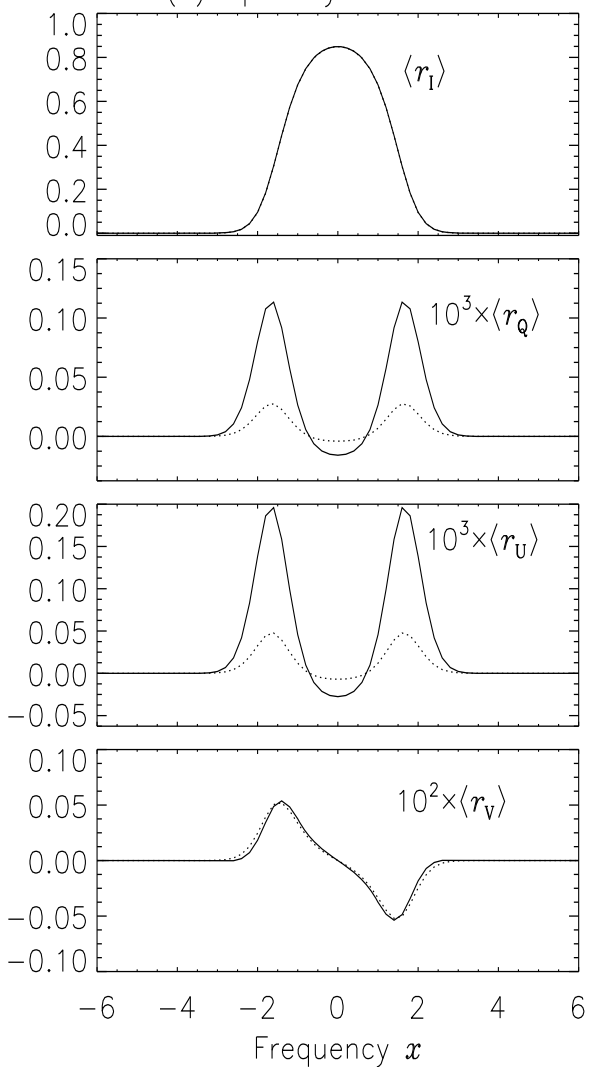

(b) Optically thick eddies
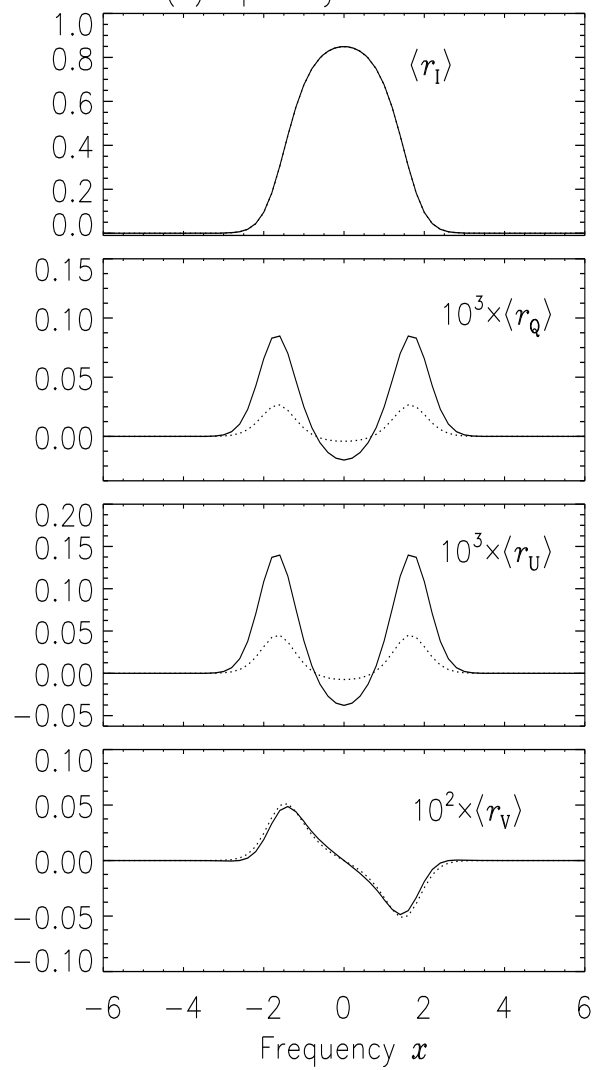

Fig. 15. Effect of turbulence on a Zeeman multiplet. Two types of PDFs are used. Solid lines: asymmetric Voigt with mean field $y_{0}=3.4$ presented as solid line in Fig. 3a. Dotted lines: asymmetric stretched exponential with mean field $y_{0}=3.2$ presented as dotted line in Fig. 8a.

Zeeman absorption matrix formally remain the same, but the normalized profiles $\varphi_{q}$ and $f_{q}$ are now given by

$\varphi_{q}=\sum_{M_{\mathrm{u}}} S_{q}\left(M_{\mathrm{l}}, M_{\mathrm{u}}\right) H\left(x_{q}, a\right)$,

and

$f_{q}=\sum_{M_{\mathrm{u}}} S_{q}\left(M_{\mathrm{l}}, M_{\mathrm{u}}\right) F\left(x_{q}, a\right)$,

respectively, subject to the constraint $M_{1}=M_{\mathrm{u}}+q$. The equations required for computing transition strengths for Zeeman multiplets and their absorption coefficients are also presented in del Toro Iniesta (2003, p. 136-145). The shapes of the Zeeman absorption matrix elements for the multiplet case are also shown in this reference.

Equations (27) and (28) are valid for a deterministic magnetic field. To use the same equations for a random field, we just need to replace $B / B_{\mathrm{D}}^{\prime}$ by $\gamma_{B}^{\prime} y$, where $y$ is defined in Eq. (2), and $\gamma_{B}^{\prime}=\Delta_{B} / B_{\mathrm{D}}^{\prime}$.

Figure 15 shows $\left\langle r_{I, Q, U, V}\right\rangle$ for a ${ }^{4} P_{1 / 2}-{ }^{4} F_{3 / 2}$ transition. For this particular transition there are two $\pi$ components and four $\sigma$ components. Keeping the same kind of spectral line as in the preceding sections (wavelength $5000 \AA$, Doppler width $1.5 \mathrm{~km} \mathrm{~s}^{-1}$ ), we find $\gamma_{B}^{\prime}=\gamma_{B} / 2=0.0028$. Our model parameters are now: $\left(a, \beta, \gamma_{B}^{\prime}\right)=(0,10,0.0028)$. For the magnetic field, we assume a fixed orientation $(\gamma, \chi)=\left(60^{\circ}, 30^{\circ}\right)$ and random strength. We use an asymmetric Voigt PDF and an asymmetric stretched exponential. They yield mean profiles $\left\langle r_{Q, U, V}\right\rangle$ with similar shapes. As always $\left\langle r_{I}\right\rangle$ remains insensitive to the choice of the PDF. $\left\langle r_{Q, U}\right\rangle$ are larger for the asymmetric Voigt PDF (solid lines), than for the asymmetric stretched exponential (dotted lines) for nearly the same values of the mean magnetic fields (3.4 and 3.2, respectively). This is because $\left\langle r_{Q, U}\right\rangle$ are sensitive to the type of PDF used, as already shown in Fig. 7 (for symmetric PDFs). $\left\langle r_{V}\right\rangle$ is generated purely by the asymmetry in the PDF, and hence it is more sensitive to the value of the mean field $y_{0}$ than to the exact shape of the PDF. For this figure we have chosen nearly the same mean value $y_{0}$ for both PDFs, with the consequence that the solid and dotted curves for $\left\langle r_{V}\right\rangle$ do not differ.

We also have calculated mean Stokes profiles for optically thick eddies. The only difference appears to be that the optically thin eddies produce $\left\langle r_{Q, U}\right\rangle$ of slightly larger magnitude than the optically thick ones.

\section{Conclusions}

We have presented mean Stokes profiles formed in media with spatially unresolved magnetic structures. We have considered the cases of optically thin and optically thick structures which involve the computation of mean absorption matrices or mean emergent Stokes profiles, respectively. We have also calculated the dispersion around the mean Stokes profiles in the optically thick limit (it is zero in the optically thin limit). The dispersion provides an upper bound for the order of magnitude of the fluctuations of the Stokes profiles around their mean values. To perform such averaging we use a probability distribution function (PDF) that describes the fluctuations of the ambient field. A Gaussian PDF with isotropic or anisotropic fluctuations has been considered in detail in Frisch et al. (2005). Here we have experimented with other types of PDFs and show the difference between the emergent mean Stokes profiles computed from them. We consider very weak fluctuations of the magnetic 
field: The Zeeman shift from the magnetic field rms fluctuations in Doppler width units, denoted here by $\gamma_{B}$, equals 0.0056 . As a consequence the $\left\langle r_{I}\right\rangle$ profiles are insensitive to the shape of the magnetic field PDF. In contrast, the other mean Stokes profiles and the dispersion are quite sensitive to the PDF shapes. For stronger fluctuations (say $\gamma_{B}=1$ ), $\left\langle r_{I}\right\rangle$ would also get significantly modified.

In Sect. 2 we consider a Voigt type PDF for the magnetic field strength derived from observations by Stenflo \& Holzreuter (2002, 2003a,b). The Voigt PDF is characterized by two parameters, the magnetic width $\Delta_{B}$ and the magnetic damping parameter $a_{B}$. For $a_{B}=0$, we recover the results of the 1D Gaussian distribution considered in Frisch et al. (2005). The effect of a non-zero $a_{B}$ on the average Stokes profiles $\left\langle r_{Q, U}\right\rangle$ is to enhance core and wing polarization. We have introduced asymmetric Voigt PDFs which provide a non-zero net magnetic flux, thereby generating an $\left\langle r_{V}\right\rangle$ profile. They are constructed by taking different magnetic damping parameters for the opposite polarities of the random field.

Stretched exponentials for the magnetic field strength derived from magneto-convection simulations by Stein \& Nordlund (2006) are considered in Sect. 3. These PDFs are characterized by a single parameter, the stretching parameter $k$, which takes values between 0 and 1 . A decrease of the stretching parameter $k$ enhances the contribution from strong fields and thus induces an increase in the values of $\left\langle r_{Q, U}\right\rangle$ (see Fig. 7). In this case also we construct asymmetric PDFs by using different values of the stretching parameter $k$ for the opposite polarities of the magnetic field.

In Sect. 4 we have examined an angular power law distribution proposed by Stenflo (1987). The only parameter for this PDF is the power law index $p$. For $p=0$, the distribution is isotropic. As $p$ increases the random field becomes more and more oriented in both the forward and backward directions. Because of the axial symmetry, $\left\langle r_{U}\right\rangle=0$ for this PDF.

For a complete description of the turbulent vector magnetic field one needs PDFs which describe both the angular and the strength distribution of the magnetic field vector. In Sect. 5 we have constructed empirical PDFs of this kind by combining a power law for the angular distribution with a Voigt function or a stretched exponential for the field strength. The PDFs for the angular and strength distributions are coupled by letting the power law index explicitly depend on the field strength. We have introduced a cut-off in the magnetic field strength below which the random magnetic field is essentially isotropic and above which it is dominantly vertical. Construction and application of these composite PDFs represent the main result of the present paper. They could be used to represent the conditions prevailing in the solar photosphere. Finally, in Sect. 6, we show how to generalize the averaging technique to the case of anomalous Zeeman splitting patterns.

Our paper highlights the need to consider fluctuations in the field orientation besides the field strength. We have experimented with a few composite PDF of this type. However, we would like observations to provide us with empirical PDFs not only for the field strength, but also for the angular distribution, but unfortunately such empirical angular PDFs are not yet available. In fact the derivation of PDFs from observations is done using "spatially resolved observations" (and not from the subtle deformations of Stokes line profiles that are produced by spatially unresolved distribution functions). It is also possible to observationally study how the PDFs depend on scale size of magnetic elements. Such spatially resolved observations provide guidance regarding the applicability of derived PDFs to the spatially unresolved domain. Thus the underlying assumption is that the PDFs derived observationally for the larger, resolved scales, can also be used for the smaller, unresolved scales.

We compute the mean Stokes profiles for a given line of sight using a PDF that is independent of depth. In reality the mean Stokes profiles are the result of space and/or time averages. Further, we have not considered realistic temperature and density stratifications, and we also ignore the velocity turbulence (except for the micro-turbulent line broadening included in the Doppler width). For these reasons our mean Stokes profiles are not yet suited for model fitting of observed Stokes profiles.

Another aspect that we need to keep in mind is the relation between the complete PDF used in our pure theoretical profiles, and the sampling that occurs in an actual observation. In our computations we use "complete" PDFs which cover the entire range in field strength and orientation. In contrast, high spatial resolution observations may only sample different fractions of a PDF in terms of strength and orientation because magnetic structures have finite sizes. A complete PDF would be obtained (asymptotically) if we add samples of many resolution elements, or if we deliberately perform a low spatial resolution measurement. Thus the observed high spatial resolution Stokes profiles represent fluctuations about a mean profile. These fluctuations will in general be of the order of the dispersion around the mean profile. The dispersion is zero in the limit of infinitely thin magnetic structures and in this limit the sampling is necessarily complete.

Stenflo \& Holzreuter (2003a) suggest that the magnetic pattern is fractal with a high degree of self-similarity over several orders of magnitude. If this coexistence of weak and strong fields continues far below the current spatial resolution limit, then the PDF sampling will be more complete than in the non-fractal case, and the sampling of the PDF may be substantial even for very high spatial resolution observations.

Acknowledgements. M.S. is financially supported by the Council of Scientific and Industrial Research (CSIR) through a Senior Research Fellowship (SRF Grant No: 9/890(01)/2004-EMR-I), which is gratefully acknowledged. M.S. is also grateful to the Indo-French Sandwich Thesis Program for making possible a visit to the Observatoire de la Côte d'Azur. Further K.N.N. and M.S. are grateful to the Laboratoire Cassiopée (CNRS), the PNST (CNRS) and the French Ministère de l'Éducation Nationale for financial support during visits at the Observatoire de la Côte d'Azur where part of this work was completed. We thank Dr. T. A. Carroll for many useful comments, which helped in improving the paper.

\section{References}

Carroll, T. A., \& Staude, J. 2003, Astron. Nachr., 324, 392

Carroll, T. A., \& Staude, J. 2005a, Astron. Nachr., 326, 296

Carroll, T. A., \& Staude, J. 2005b, ESA SP-596: Chromospheric and Coronal Magnetic Fields, ed. D. E. Innes, A. Lagg, \& S. K. Solanki, 30 August 2005, Katlenburg-Lindau, Germany, published on CDROM, 9.1

Carroll, T. A., \& Staude, J. 2006, Solar Polarization 4, ed. R. Casini, \& B. W. Lites, 19 September 2005, National Center for Atmospheric Research, Boulder, Colorado, USA, ASP Conf. Ser., 358, 137

Carroll, T. A., \& Kopf, M. 2007, A\&A, 468, 323

del Toro Iniesta, J. C. 2003, Introduction to Spectro polarimetry (Cambridge University Press)

Dolginov, A. Z., \& Pavlov, G. G. 1972, Soviet Ast., 16, 450 (transl. from Astron. Zh., 49, 555)

Domínguez Cerdeña, I., Sánchez Almeida, J., \& Kneer, F. 2006, ApJ, 636, 496 Domke, H., \& Pavlov, G. G. 1979, Ap\&SS, 66, 47

Frisch, H., Sampoorna, M., \& Nagendra, K. N. 2005, A\&A, 442, 11

Frisch, H., Sampoorna, M., \& Nagendra, K. N. 2006a, A\&A, 453, 1095

Frisch, H., Sampoorna, M., \& Nagendra, K. N. 2006b, Solar Polarization 4, ed. R. Casini, \& B. W. Lites, 19 September 2005, National Center for Atmospheric Research, Boulder, Colorado, USA, ASP Conf. Ser., 358, 126 
Frisch, H., Sampoorna, M., \& Nagendra, K. N. 2007, Mem. S. A. It., 78, 142 Landi Degl'Innocenti, E., 1994, Solar Surface Magnetism, ed. R. J. Rutten, \&

C. J. Schrijver (Dordrecht: Kluwer Academic Publishers), 29

Landi Degl'Innocenti, E., \& Landolfi, M. 2004, Polarization in Spectral Lines (Dordrecht: Kluwer Academic Publishers)

Lites, B. W., Socas-Navarro, H., Kubo, M., et al. 2007, PASJ, 59, S571

Lites, B. W., Kubo, M., Socas-Navarro, H., et al. 2008, ApJ, 672, 1237

Mihalas, D. 1978, Stellar Atmospheres, 2nd edn. (San Francisco: Freeman)

Orozco Suárez, D., Bellot Rubio, L. R., del Toro Iniesta, J. C., et al. 2007, ApJ, 670, L61

Rachkovsky, D. N., 1962a, Izv. Krymsk. Astrofiz. Obs., 27, 148

Rachkovsky, D. N., 1962b, Izv. Krymsk. Astrofiz. Obs., 28, 259

Sampoorna, M., Frisch, H., \& Nagendra, K. N. 2008, New Ast., 13, 233

Sánchez Almeida, J., Landi Degl'Innocenti, E., Martinez Pillet, V., \& Lites, B. W. 1996, ApJ, 466, 537

Stein, R. F., \& Nordlund, A. 2006, ApJ, 642, 1246

Stenflo, J. O. 1971, Solar Magnetic Fields, ed. R. Howard, IAU Coll., 43, 101
Stenflo, J. O. 1973, Sol. Phys., 32, 41

Stenflo, J. O. 1987, Sol. Phys., 114, 1

Stenflo, J. O. 1994, Solar Magnetic Fields (Dordrecht: Kluwer Academic Publishers)

Stenflo, J. O., \& Holzreuter, R. 2003a, Current Theoretical models and Future High Resolution Solar Observations: Preparing for ATST, ed. A. A. Pevtsov, \& H. Uitenbroek, ASP Conf. Ser., 286, 169

Stenflo, J. O., \& Holzreuter, R. 2003b, Astron. Nachr., 324, No.4, 397

Stenflo, J. O., \& Holzreuter, R. 2002, ESA SP-505: Magnetic coupling of the Solar Atmosphere, ed. H. Sawaya-Lacoste, 101

Stenflo, J. O., \& Lindegren, L. 1977, A\&A, 59, 367

Stenflo, J. O., Harvey, J. W., Brault, J. W., \& Solanki, S. 1984, A\&A, 131, 333

Unno, W. 1956, PASJ, 8, 108

Unno, W. 1959, ApJ, 129, 375

Varshalovich, D. A., Moskalev, A. N., \& Khersonskii, V. K. 1988, Quantum Theory of Angular Momentum (World Scientific)

Vögler, A., Shelyag, S., Schüssler, M., et al. 2005, A\&A, 429, 335 